\title{
ON THE IRREDUCIBILITY OF IRREDUCIBLE CHARACTERS OF SIMPLE LIE ALGEBRAS
}

\begin{abstract}
C. S. RAJAN
ABSTRACT. We establish an irreducibility property for the characters of finite dimensional, irreducible representations of simple Lie algebras (or simple algebraic groups) over the complex numbers, i.e., that the characters of irreducible representations are irreducible after dividing out by (generalized) Weyl denominator type factors.

For $S L(r)$ the irreducibility result is the following: let $\lambda=\left(a_{1} \geq a_{2} \geq\right.$ $\cdots \geq a_{r-1} \geq 0$ ) be the highest weight of an irreducible rational representation $V_{\lambda}$ of $S L(r)$. Assume that the integers $a_{1}+r-1, a_{2}+r-2, \cdots, a_{r-1}+1$ are relatively prime. Then the character $\chi_{\lambda}$ of $V_{\lambda}$ is strongly irreducible in the following sense: for any natural number $d$, the function $\chi_{\lambda}\left(g^{d}\right), g \in S L(r, \mathbb{C})$ is irreducible in the ring of regular functions of $S L(r, \mathbb{C})$.
\end{abstract}

\section{INTRODUCTION}

In $[\mathrm{R}$, the following unique factorization property of tensor products of irreducible representations of a complex simple Lie algebra $\mathfrak{g}$ was proved:

Theorem 1.1. Let $V_{1}, \cdots, V_{n}, W_{1}, \cdots, W_{m}$ be non-trivial irreducible representations of $\mathfrak{g}$ (resp. rational irreducible representations of $G L(r)$ for $r \geq 2$ ) such that

$$
V_{1} \otimes \cdots \otimes V_{n} \simeq W_{1} \otimes \cdots \otimes W_{m}
$$

as $\mathfrak{g}$ (resp. $G L(r)$ )-modules. Then $n=m$, and there is a permutation $\sigma$ of $\{1, \cdots, n\}$ such that $V_{i} \simeq W_{\sigma(i)}$ (resp. $V_{i} \simeq W_{\sigma(i)} \otimes \operatorname{det}^{k_{i}}$ for some integers $k_{i}$ ).

In this introduction we restrict ourselves to $G L(r)$. Given a rational representation $V$ of $G L(r)$, let

$$
\chi_{V}(g)=\operatorname{Tr}(V(g)), \quad g \in G L(r),
$$

denote the character of $V$. Since the character determines the representation up to isomorphism, the hypothesis of the foregoing theorem can be reformulated as an equality of products of characters:

$$
\chi_{V_{1}} \cdots \chi_{V_{n}}=\chi_{W_{1}} \cdots \chi_{W_{m}} .
$$

Now, the character is a regular $G L(r)$-invariant function of $G L(r)$. The unique decomposition of tensor products will follow if the non-trivial characters of irreducible rational representations of $G L(r), r \geq 2$, are irreducible in the ring of invariant regular functions on $G L(r)$.

But this turns out to be manifestly false for $G L(2)$, where the characters restricted to the diagonal torus are given up to powers of $x_{1} x_{2}$ by cyclotomic type

Received by the editors August 17, 2012 and, in revised form, January 15, 2013.

2010 Mathematics Subject Classification. Primary 17B10; Secondary 20G05. 
homogeneous polynomials in two variables of the form $\left(x_{1}^{a+1}-x_{2}^{a+1}\right) /\left(x_{1}-x_{2}\right)$, hence factorizable over $\mathbb{C}$.

Up to twisting by a power of the determinant, we can assume that the irreducible representations $V_{\lambda}$ of $G L(r)$ are parametrized by their highest weights,

$$
\lambda=\left(a_{1}, \cdots a_{r}\right), a_{1} \geq a_{2} \geq \cdots \geq a_{r}=0,
$$

where $a_{i}$ are non-negative integers. Let $T$ denote the diagonal torus of $G L(r)$ consisting of diagonal matrices with diagonal entries given by $x=\left(x_{1}, \cdots, x_{r}\right)$. Let $W$ denote the Weyl group of $(G L(r), T)$, the symmetric group on $r$-variables, acting by permutations on $T$. Restricting to the torus $T$ gives an isomorphism of the algebra of conjugacy invariant regular functions on $G L(r)$ onto the algebra of Weyl group invariant regular functions on $T$; hence we can consider the characters restricted to $T$. Let $\epsilon: W \rightarrow \mathbb{Z} / 2 \mathbb{Z}$ be the sign homomorphism. For a weight $\mu=$ $\left(b_{1}, \cdots, b_{r}\right)$ with $b_{1}, \cdots, b_{r}$ non-negative integers, define the Schur-Weyl function $S(\mu)(x)$ by

$$
S(\mu)(x)=\sum_{\sigma \in W} \epsilon(\sigma) x_{1}^{b_{\sigma(1)}} \cdots x_{r}^{b_{\sigma(r)}}=\operatorname{det}\left(x_{i}^{b_{j}}\right) .
$$

This is an alternating polynomial in variables $x_{1}, \cdots, x_{r}$, which vanishes if $b_{i}=b_{j}$ for a pair of indices $i \neq j$. Let

$$
\rho=(r-1, r-2, \cdots, 0)
$$

be the 'Weyl weight'. The Weyl denominator function $S(\rho)(x)$ is the Vandermonde determinant and has a product decomposition,

$$
S(\rho)(x)=\prod_{i<j}\left(x_{i}-x_{j}\right) .
$$

Given any weight $\mu$ of $G L(r)$, it is easy to observe that the Weyl denominator $S(\rho)$ divides $S(\mu)$ in the polynomial ring in $r$-variables. The Schur-Weyl character formula for $G L(r)$ gives the restriction of the character $\chi_{\lambda}$ of $V_{\lambda}$ to the torus $T$ by the formula

$$
\begin{aligned}
\chi_{\lambda}(x) & =S(\lambda+\rho)(x) / S(\rho)(x) \\
& =\operatorname{det}\left(x_{i}^{a_{j}+r-j}\right) / \operatorname{det}\left(x_{i}^{r-j}\right), \quad x \in T .
\end{aligned}
$$

Based on the factorization of characters for $G L(2)$ governed by cyclotomic theory, the initial attempts in the general case towards finding the irreducibility property of characters underlying the unique factorization theorem was to look for divisibility relations amongst the irreducible characters parametrized by appropriate 'divisibility properties' amongst the highest weights. Now let $\lambda=\left(a_{1}>\cdots>a_{r-1}>a_{r}=\right.$ 0 ) be a dominant regular weight for $G L(r)$. Denote by $d(\lambda)$ the greatest common divisor of the integers $a_{i}$ :

$$
d(\lambda):=\operatorname{gcd}\left(a_{1}, \cdots, a_{r}\right) .
$$

We observe that the Schur-Weyl sum satisfies the 'scaling' relation,

$$
S(d \lambda)\left(x_{1}, \cdots, x_{r}\right)=S(\lambda)\left(x_{1}^{d}, \cdots, x_{r}^{d}\right),
$$

where $d \lambda=\left(d a_{1}, \cdots, d a_{r}\right)$. Combined with the divisibility relation $S(\rho) \mid S(\lambda)$ for any weight $\lambda$, this implies the divisibility relation,

$$
S(d \rho) \mid S(\lambda) \quad \text { if } \quad d \mid d(\lambda) .
$$


After a few calculations for $G L(3)$, it turns out that apart from the obvious divisibilty relation given by equation (1.4), other divisibility relations are hard to come by. If $\lambda$ is not a multiple of $\rho$, define $C(\lambda)$ as the quotient,

$$
C(\lambda)=S(\lambda) / S(d(\lambda) \rho) .
$$

This defines a symmetric polynomial in $r$-variables. The experimental observations for $G L(3)$ leads us to expect and prove the following theorem:

Theorem 1.2. Let $\lambda=\left(a_{1}, \cdots a_{r}\right), a_{1}>a_{2}>\cdots>a_{r}=0$ be a dominant, regular integral weight for $G L_{r}$. If $\lambda$ is not a multiple of $\rho$, viz., $\lambda \neq d(\lambda) \rho$, then $C(\lambda)$ is absolutely irreducible, i.e., irreducible in the ring $\mathbb{C}\left[x_{1}, \cdots, x_{r}\right]$.

This theorem has been proved R. Dvornicich and U. Zannier [DZ]. However their motivation and proof are completely different.

A uniqueness result can also be established (see Theorem 2.4), that $\lambda$ can be recovered from $C(\lambda)$ as long as $C(\lambda) \neq 1$. As a corollary one gets a proof of Theorem 1.1

We now give a brief indication of the proof of Theorem 1.2 and refer to Section 5 for further details. The proof proceeds by induction on $r$. The inductive step is carried out using the cofactor expansion of the determinant expression for $S(\lambda)$. The cofactor expansion gives us a polynomial in one variable with coefficients that are again of the form $S(\mu)$ with $\mu$ a weight for $G L(r-1)$.

There are three parts to the proof. The heart of the proof is the following (see Proposition 5.1): suppose $\mu, \eta$ are dominant regular weights for $G L(r-1)$ with $\eta=\mu+(c, 0, \cdots, 0)$ for some natural number $c$ such that both $d(\mu)$ and $d(\eta)$ are divisible by a natural number $d$. Assume further that there exist non-monomial symmetric polynomials $U, V$ and polynomials $X, Y$ satisfying the following system of equations:

$$
U V=S(\mu) / S(d \rho) \quad \text { and } \quad U X+V Y=S(\eta) / S(d \rho) .
$$

Then the conclusion is that $(d(\mu), d(\eta))>d$. The proof of this proposition uses some arithmetical ideas when $r=3$.

For example, let $r=3$ and $\lambda=\left(a_{1}>a_{2}>0\right)$ be a regular weight for $G L(3)$, with gcd $\left(a_{1}, a_{2}\right)=d$. Consider a factorization $C(\lambda)=Q R$ such that the leading coefficients $U$ and $V$ respectively of $Q$ and $R$ considered as a polynomial in the variable $x_{1}$ with coefficients polynomials in $x_{2}, x_{3}$ are not monomials. A little argument using divisibility as used in the proof of Eisenstein criterion, yields a pair of equations of the form

$$
U V=\left(x_{2}^{a_{2}}-x_{3}^{a_{2}}\right) /\left(x_{2}^{d}-x_{3}^{d}\right), \quad U X+V Y=\left(x_{2}^{a_{1}}-x_{3}^{a_{1}}\right) /\left(x_{2}^{d}-x_{3}^{d}\right),
$$

for some polynomials $X$ and $Y$. Proposition 6.1, the specialization of Proposition 5.1. applies to give a contradiction. For higher ranks, the key observation to the proof of Proposition 5.1 is to realize that an inductive proof is possible; this in turn depends crucially on the fact that the Weyl denominator $S(\rho)$ divides any $S(\lambda)$.

The rest of the proof is built around this proposition. A use of the Eisenstein criterion allows us to rule out symmetric 'monic' factorizations, i.e., symmetric factorizations $C(\lambda)=U_{0} V_{0}$ such that either both the leading or both the constant coefficients of $U_{0}$ and $V_{0}$ (with respect to the 'cofactor expansion' expressing them as a polynomial in $x_{1}$ with coefficients polynomials in the $r-1$ variables $x_{2}, \cdots, x_{r}$ ) are non-monomial (see Proposition 5.2 and Proposition 8.1). 
Finally, one reduces a non-symmetric factorization to either of the above propositions.

The outline of this paper is as follows: in the next section, we state the theorem for a general simple Lie algebra, where we consider the characters as elements in the algebra of the Weyl group invariants of the group algebra of the weight lattice. Extra complications arise in the non-simply laced cases. The expected analogue of Theorem 1.2 however is not proved in general in this paper, and there is a gap in the proof for a subclass of weights for $G_{2}$ and $F_{4}$.

In Section 3, the irreducibility theorem is stated in the context of regular functions of a simple algebraic group, and a proof is given assuming the statement for the Lie algebra. In Section 4 we recall the technique of cofactor expansions, which allows an inductive setup based on the rank for the proof of the irreducibility result.

Section 5 contains the statements of the key propositions and a proof of the main Theorem 2.3 assuming the validity of these propositions. The overall idea of the proof is the same as that of $G L(r)$; in fact, it is simpler in some places for the Lie algebras of types $D$ and $E$. However, both the statement and the proofs of the various propositions are more complicated for the non-simply laced Lie algebras.

Section 6 gives a proof of the key Proposition 5.1 for the root system $s l_{2}$ using some facts from arithmetic. The whole proof emanates from this proposition, and how it helps in establishing the irreducibility property for $G L(3)$ as explained above.

Section 7 gives a proof of Proposition 5.1 in the general case by an inductive argument, a 'swindle' using the universal divisibility of the Weyl denominator.

The proof of Proposition 5.2 ruling out the existence of invariant monic factorizations is given in Section 8 . This is an application of the method of proof used in the classical Eisenstein criterion for irreducibility of polynomials. It is here that a gap arises in the proof for a subclass of weights for $G_{2}$ and $F_{4}$.

The uniqueness result Theorem 2.4 is proved in Section 9. This section also contains some preliminary results used in the proofs of the various propositions: for example, in the case of $G L(r)$, the fact that $S(\lambda)$ is separable for $G L(s)$ with $s<r$. In this case, it is quite easy to observe this fact assuming Theorem 1.2 and the factorization of $S(d \rho)$.

Finally, in Section 10 we extend the proof of the irreducibility result for $G L(r)$ from the ring of symmetric functions in $r$-variables to the polynomial ring in $r$ variables.

\section{Simple Lie ALGEBRAS}

In this section, we consider the general case of a simple Lie algebra $\mathfrak{g}$ over $\mathbb{C}$, fix the notation and state the main irreducibility theorem, which requires that it be modified when the Lie algebra has at least two roots of different lengths.

2.1. Notation. We first fix the notation and recall relevant facts from the theory of root systems (see $[\mathrm{B},[\mathrm{H}]$ ).

Let $\mathfrak{g}$ be a simple Lie algebra over $\mathbb{C}$, and let $\mathfrak{h}$ be a Cartan subalgebra of $\mathfrak{g}$. Denote by $\Phi \subset \mathfrak{h}^{*}$ the roots of the pair $(\mathfrak{g}, \mathfrak{h})$, and let $E$ be the real subspace of $\mathfrak{h}^{*}$ generated by $\Phi$. The dual of the restriction of the Killing form to $\mathfrak{h} \times \mathfrak{h}$ defines a non-degenerate symmetric bilinear form on $E$. With respect to this inner product, $\Phi$ defines a root system in $E$.

Denote by $\Phi^{+} \subset \Phi$ (resp. $\left.\Delta \subset \Phi^{+} ; \Phi^{*} \subset E^{*} ; \Phi^{*+} ; \Delta^{*}\right)$ the subset of positive roots with respect to some ordering of the root system (resp. a base for $\Phi^{+}$; the set 
of coroots; positive coroots; simple coroots). Given a root $\alpha \in \Phi, \alpha^{*}$ will denote the corresponding coroot.

Denote by $\langle.,\rangle:. E^{*} \times E \rightarrow \mathbb{R}$ the duality pairing. For any root $\alpha$, we have $\left\langle\alpha^{*}, \alpha\right\rangle=2$, and the pairing takes values in integers when the arguments consist of roots and coroots.

Let $W\left(\right.$ resp. $\left.W^{*}\right)$ denote the Weyl group of the (resp. dual) root system. The Weyl group $W\left(\right.$ resp. $\left.W^{*}\right)$ is the subgroup of $\operatorname{Aut}(E)\left(\operatorname{resp} . \operatorname{Aut}\left(E^{*}\right)\right)$ generated by the reflections $s_{\alpha}$ of $E$ (resp. $s_{\alpha^{*}}$ of $E^{*}$ ) defined by

$$
s_{\alpha}(u)=u-\left\langle\alpha^{*}, u\right\rangle \alpha \text { and } s_{\alpha^{*}}(x)=x-\langle x, \alpha\rangle \alpha^{*},
$$

where $x \in E^{*}$ and $u \in E$. We have $s_{\alpha}(\Phi) \subset \Phi$ and $s_{\alpha^{*}}\left(\Phi^{*}\right) \subset \Phi^{*}$. There is a natural isomorphism between the Weyl groups of the root system and the dual root system, given by $\alpha \mapsto \alpha^{*}$ and $s_{\alpha^{*}}={ }^{t} s_{\alpha}$, the transpose of $s_{\alpha}$. We identify the two actions of the Weyl group.

Remark 2.1. Sometimes we formulate the propositions for a based root system $\mathcal{R}=(E, \Phi, \Delta)$ instead of $\mathfrak{g}$, and at times we refer to the root system just by $\Phi$.

Let $l(w)$ denote the length of an element in the Weyl group, given by the least length of a word in the $s_{\alpha}, \alpha \in \Delta$, defining $w$. Let $\epsilon(w)=(-1)^{l(w)}$ be the sign character of $W$.

Denote by $P \subset E$ (resp. $P^{+} \subset P, P^{++} \subset P_{+}, P^{*} \subset E^{*}, P^{*,++}$ ) the lattice of integral weights (resp. dominant integral weights, dominant regular weights, integral co-weights, dominant regular coweights). Let $r=|\Delta|$ be the rank of $\Phi$. For a simple root $\alpha \in \Delta$, denote by $\omega_{\alpha}$ (resp. $\omega_{\alpha}^{*}$ ) the corresponding fundamental weight (resp. coweight) defined by

$$
\left\langle\beta^{*}, \omega_{\alpha}\right\rangle=\delta_{\alpha \beta} \quad \text { and } \quad\left\langle\omega_{\alpha}^{*}, \beta\right\rangle=\delta_{\alpha \beta}, \quad \alpha, \beta \in \Delta .
$$

The fundamental weights form a $\mathbb{Z}$-basis for $P$. A weight $\lambda$ can be expressed as a sum,

$$
\lambda=\sum_{\alpha \in \Delta} m_{\alpha}(\lambda) \omega_{\alpha}
$$

where $m_{\alpha}(\lambda)=\left\langle\alpha^{*}, \lambda\right\rangle$ are the coefficients of $\lambda$ with respect to the basis of $P$ determined by $\Delta$. The weight $\lambda$ is regular (resp. dominant) if for any $\alpha \in \Delta$, $m_{\alpha}(\lambda) \neq 0$ (resp. $m_{\alpha}(\lambda) \geq 0$ ).

2.2. Schur-Weyl elements. For any ring $A$ and commutative group $X$, let $A[X]$ denote the group algebra of $X$ with coefficients in $A$. We work with multiplicative (exponential) notation. A basis for $A[X]$ is given by the elements indexed by $e^{x}$ for $x \in A$. The group law is expressed by $e^{x} \cdot e^{y}=e^{x+y}, x, y \in X$. The action of the Weyl group is seen as $w e^{\lambda}=e^{w \lambda}$, where $w \lambda=w(\lambda)$.

For a weight $\lambda \in P$, define the Schur-Weyl element $S(\lambda) \in \mathbb{Z}[P]$ as

$$
S(\lambda)=\sum_{w \in W} \epsilon(w) e^{w \lambda} .
$$

The Schur-Weyl elements are alternating with respect to the action of the Weyl group $W$ on $\mathbb{Z}[P]$,

$$
\sigma(S(\lambda))=\epsilon(\sigma) S(\lambda), \quad \sigma \in W .
$$

The group algebra $A[P]$ can be identified with a Laurent polynomial ring in $r$-variables over $A$, where $r=\operatorname{dim}_{\mathbb{R}}(E)$ is the rank of $\mathfrak{g}$. In particular, $\mathbb{C}[P]$ is a unique factorization domain. 
2.3. Weyl character formula. Let $V$ be a finite dimensional $\mathfrak{g}$-module. With respect to the action of $\mathfrak{h}$, there is a decomposition,

$$
\begin{gathered}
V=\bigoplus_{\pi \in P} V^{\pi}, \\
\text { where } V^{\pi}=\{v \in V \mid X v=\pi(X) v, X \in \mathfrak{h}\}
\end{gathered}
$$

are the weight spaces of $V$. The linear forms $\pi$ for which $V^{\pi}$ are non-zero are the weights of $V$, and $V^{\pi}$ is the subspace of $V$ consisting of eigenvectors of $H$ with weight $\pi$. The formal character $\chi_{V} \in \mathbb{Z}[P]$ of $V$ is defined as

$$
\chi_{V}=\sum_{\pi \in P} m(\pi) e^{\pi}
$$

where $m(\pi)=\operatorname{dim}\left(V^{\pi}\right)$ is the multiplicity of $\pi$. The character is invariant under the action of the Weyl group.

The irreducible finite dimensional $\mathfrak{g}$-modules are indexed by elements in $\lambda \in P^{+}$, given by Cartan-Weyl theory. To each dominant, integral weight $\lambda$, we denote the corresponding irreducible $\mathfrak{g}$-module with highest weight $\lambda$ by $V_{\lambda}$ and the formal character of $V_{\lambda}$ by $\chi_{\lambda}$. The Weyl character formula gives the formula for the formal character $\chi_{\lambda}$ :

$$
\text { Weyl character formula: } \chi_{\lambda}=S(\lambda+\rho) / S(\rho),
$$

where $\rho$ is the Weyl weight defined by the equations

$$
\rho=\frac{1}{2} \sum_{\alpha \in \Phi^{+}} \alpha=\sum_{\alpha \in \Delta} \omega_{\alpha} .
$$

Remark 2.2. It can be seen as an application of the Weyl character formula (or directly by induction) that for $\lambda \in P^{++}$, the element $S(\lambda)$ is non-vanishing.

2.4. Divisibility. For any weight $\lambda$, define

$$
d(\lambda)=\operatorname{gcd}\left\{m_{\alpha}(\lambda) \mid \alpha \in \Delta\right\} .
$$

Equivalently, $d(\lambda)$ can be defined as the largest integer $d$ for which $d \rho$ divides $\lambda$ (a regular weight $\mu$ is said to divide a weight $\lambda$ if for every $\alpha \in \Delta$, the coefficient $m_{\alpha}(\mu)$ divides $\left.m_{\alpha}(\lambda)\right)$.

Example 2.1. For $S L(r)$, denote by $\omega_{i}, 1 \leq i \leq r-1$, the set of fundamental weights given by the highest weights of the $i$-th exterior power of the natural representation of $S L(r)$. For $\lambda=\left(a_{1}, a_{2}, \cdots, a_{r}\right)$ a weight of $G L(r)$, the coefficients are given by $m_{i}(\lambda)=a_{i}-a_{i+1}$. If the weight $\lambda$ is normalized so that $a_{r}=0$, then the definition of $d(\lambda)$ given in the previous section agrees with the above definition.

The following proposition, especially the divisibility aspect, is of fundamental importance to us in this paper, and is needed in the formulation as well as the proof of the main theorem (in establishing the inductive argument in the proof of Proposition 5.1. 
Proposition 2.1. (a) (Factorization of a Weyl denominator) For any positive integer $d$, there is a factorization in the Laurent polynomial ring $\mathbb{Z}\left[\frac{1}{2} P\right]$,

$$
\begin{aligned}
S(d \rho) & =\prod_{\alpha \in \Phi^{+}}\left(e^{d \alpha / 2}-e^{-d \alpha / 2}\right) \\
& =e^{-d \rho} \prod_{\alpha \in \Phi^{+}}\left(e^{d \alpha}-1\right) .
\end{aligned}
$$

(b) (Divisibility by a Weyl denominator) For any weight $\lambda \in P$ with greatest common divisor $d(\lambda)$ and any natural number $d$ dividing $d(\lambda)$, the element $S(d \rho)$ divides $S(\lambda)$ in $\mathbb{Z}[P]^{W}$.

(c) (Separability of a Weyl denominator) The generalized Weyl denominators $S(d \rho)$ are separable elements in the ring $\mathbb{C}[P]$.

Proof. Part (a) is well known. For $d=1$, (b) is a restatement of [B. Proposition 2, Chapter VI, Section 3.3, page 185]. For any natural number $d$, there are the 'scaling' maps $[d]: \mathbb{Z}[P] \rightarrow \mathbb{Z}[P]$ induced by multiplication by $d$ on $P$. One has

$$
[d](S(\lambda))=S(d \lambda) .
$$

Part (b) now follows from the 'universal' divisibility that $S(\rho)$ divides $S(\lambda)$.

A proof of (c) is given later as Corollary 9.1 in Section 9 .

2.5. Duality. It was pointed out by P. Deligne that for a general simple Lie algebra, there are extra factors which arise whenever there are at least two roots of different lengths in a root system for $\mathfrak{g}$. These extra factorizations arise from duality: the weight lattices of the root system and its dual are seen to be commensurable lattices in $E$ as $W$-modules. Normalizing the square of the length of the shorter root to be 2 , this can be seen by the standard identification $\alpha^{*}=2 \alpha /(\alpha, \alpha)$. If the root system is not simply laced, then these lattices are not isomorphic. This gives rise to new factorizations, as the Weyl denominator weights $\rho$ and $\rho^{*}$ are not rational multiples of each other.

Let $\alpha_{l}$ (resp. $\left.\alpha_{s}\right)$ be a long (resp. short) root in $E$. Define

$$
m(\Phi)=\left(\alpha_{l}, \alpha_{l}\right) /\left(\alpha_{s}, \alpha_{s}\right) .
$$

The classification of root systems implies that the possible values of $m(\Phi)$ are

$$
m(\Phi)= \begin{cases}1 & \text { if } \Phi \text { is of type } A, D, E \\ 2 & \text { if } \Phi \text { is of type } B, C, F \\ 3 & \text { if } \Phi \text { is of type } G .\end{cases}
$$

The map

$$
\alpha^{*} \mapsto 2 \alpha /(\alpha, \alpha)
$$

provides a $W$-equivariant identification of the coweight lattice $P^{*}$ with a lattice in $E$ (for the extended action of $W$ on $E$ ).

We normalize the inner product by requiring that the short root have the square of its length as 2. Via this identification, we get

$$
\omega_{\alpha}^{*}= \begin{cases}\omega_{\alpha} & \text { if } \alpha \text { is a short root } \\ \omega_{\alpha} / m(\Phi) & \text { if } \alpha \text { is a long root. }\end{cases}
$$


We identify $P^{*}$ with its image in $E$. The lattices $P$ and $P^{*}$ are commensurable in E. Let

$$
\tilde{P}=m(\Phi) P^{*} .
$$

The lattice $\tilde{P}$ can be thought of as a weight lattice of the the 'normalized' dual root system, where the short roots in $\mathcal{R}^{*}$, which correspond to the long roots in $\mathcal{R}$, have squared length 2 . Let $\rho^{*}$ denote the Weyl weight of the dual root system defined by $\Phi^{*}$. Define

$$
\tilde{\rho}=m(\Phi) \rho^{*}=\sum_{\alpha \in \Delta_{s}} m(\Phi) \omega_{\alpha}+\sum_{\alpha \in \Delta_{l}} \omega_{\alpha}
$$

where $\Delta_{s}=\Delta \cap \Phi_{s}$ (resp. $\Delta_{l}=\Delta \cap \Phi_{l}$ ) and $\Phi_{s}$ (resp. $\Phi_{l}$ ) is the subset of $\Phi$ consisting of the short (resp. long) roots. From the formula for $m(\Phi)$, it follows that $\tilde{\rho}=\rho$ precisely for the simply laced root systems (of type $A, D, E$ ). It can be seen that the element $\tilde{\rho}$ is a generator of the group $\mathbb{Q} \rho^{*} \cap P$.

The Weyl group invariance of the pairing allows us to transfer the factorization and divisibility relations for the Schur-Weyl sums of integral multiples of $\rho^{*}$ in the group ring $\mathbb{Z}\left[P^{*}\right]$ to the group ring $\mathbb{Z}[P]$ of the lattice $P$. The point is that when there are at least two roots having different lengths, this gives us new factorizations and divisibility relations. This is expressed by the following proposition which is 'dual' to Proposition 2.1

Proposition 2.2. (a) (Factorization of a dual Weyl denominator) For any positive integer $d$, there is a factorization in the Laurent polynomial ring $\mathbb{Z}\left[\frac{1}{2} P\right]$,

$$
\begin{aligned}
S(d \tilde{\rho}) & =\prod_{\alpha \in \Phi_{s}^{+}}\left(e^{d m(\Phi) \alpha / 2}-e^{-d m(\Phi) \alpha / 2}\right) \prod_{\alpha \in \Phi_{l}^{+}}\left(e^{d \alpha / 2}-e^{-d \alpha / 2}\right) \\
& =e^{-d \tilde{\rho}} \prod_{\alpha \in \Phi_{s}^{+}}\left(e^{d m(\Phi) \alpha}-1\right) \prod_{\alpha \in \Phi_{l}^{+}}\left(e^{d \alpha}-1\right) .
\end{aligned}
$$

(b) (Divisibility by a dual Weyl denominator) If $d \tilde{\rho} \mid \lambda$, then $S(d \tilde{\rho})$ divides $S(\lambda)$ in $\mathbb{Z}[P]$.

(c) (Separability of a dual Weyl denominator) The elements $S(d \tilde{\rho})$ are separable in the ring $\mathbb{C}[P]$.

We refer to elements of the form $S(d \rho)$ or $S(d \tilde{\rho})$ as elements of (generalized) Weyl denominator type in $\mathbb{Z}[P]$.

2.6. Factors of $S(\lambda)$ of Weyl denominator type. For $\lambda \neq \rho$ a dominant regular weight in $P^{++}$, define

$$
D(\lambda)=\operatorname{lcm}_{d \rho \mid \lambda,} \underset{e \tilde{\rho} \mid \lambda, \quad e \tilde{\rho} \neq \lambda}{ }(S(d \rho), S(e \tilde{\rho})),
$$

where the lcm is taken in the Laurent polynomial ring $\mathbb{Z}[P]^{W}$. By $[\mathrm{B}$, Theorem 1 , Ch. VI, Section 4 , page 188$]$, the ring $\mathbb{Z}[P]^{W}$ is isomorphic to a polynomial ring over $\mathbb{Z}$ in $r$-variables:

$$
\mathbb{Z}[P]^{W} \simeq \mathbb{Z}\left[\left\{\omega_{\alpha} \mid \alpha \in \Delta\right\}\right] .
$$

Hence the units of the ring $\mathbb{Z}[P]^{W}$ are isomorphic to $\{1,-1\}$. With respect to the dominant order (a weight is non-negative if it can be written as a non-negative linear combination of positive roots), the leading term of $S(\lambda)$ is given by $e^{\lambda}$. The least common multiple in the definition of $D(\lambda)$ in the above equation is taken to 
be the element whose coefficient of the leading monomial occuring in $D(\lambda)$ with respect to the dominant ordering is positive (equal to 1). Define

$$
C(\lambda)=S(\lambda) / D(\lambda)
$$

to be the quotient of the Schur-Weyl sum $S(\lambda)$ divided by the obvious factors arising from the Weyl character formula and duality. Since $S(\lambda)$ is alternating, it follows that $C(\lambda) \in \mathbb{Z}[P]^{W}$.

We now look at the structure of $D(\lambda)$. We have the following inclusions:

$$
P^{*} \supset P \supset \tilde{P}=m(\Phi) P^{*} \supset m(\Phi) P .
$$

Given $\lambda \in \tilde{P}$, define $\tilde{d}(\lambda)$ as the largest integer $d$ for which $d \tilde{\rho}$ divides $\lambda$.

Lemma 2.1. Suppose that $\lambda \in P^{++}$is neither a multiple of $\rho$ or $\tilde{\rho}$. Then

$$
D(\lambda)= \begin{cases}S(d(\lambda) \rho), & \text { if } \lambda \in m(\Phi)^{i} P \backslash m(\Phi)^{i+1} P^{*} \text { for some } i \geq 0, \\ S(\tilde{d}(\lambda) \tilde{\rho}), & \text { if } \lambda \in m(\Phi)^{i+1} P^{*} \backslash m(\Phi)^{i+1} P \text { for some } i \geq 0 .\end{cases}
$$

Proof. An element $\lambda \in m(\Phi)^{i} P \backslash m(\Phi)^{i+1} P^{*}$ for some $i \geq 0$ iff $m(\Phi)^{i} \mid m_{\alpha}(\lambda), \forall \alpha \in$ $\Delta$ and there exists an $\alpha \in \Delta_{s}$ such that $m(\Phi)^{i+1}$ does not divide $m_{\alpha}(\lambda)$. If $i=0$, then $\tilde{\rho}$ does not divide $\lambda$. For $i>0$, suppose $e m(\Phi)^{j} \tilde{\rho}$ divides $\lambda$, with $e$ coprime to $m(\Phi)$. This implies that $e m(\Phi)^{j+1} \mid m_{\alpha}(\lambda)$ for all $\alpha \in \Delta_{s}$. Hence, $j \leq i-1$, and it follows that $e m(\Phi)^{j+1} \rho \mid \lambda$. But $e m(\Phi)^{j} \tilde{\rho}$ divides $e m(\Phi)^{j+1} \rho$. Thus the lcm can be taken amongst factors of the form $S(f \rho)$ with $f \rho \mid \lambda$. Since $\lambda$ is not a multiple of $\rho$, the lcm is given by $S(d(\lambda) \rho)$, and this proves the first case.

In the second case, an element $\lambda \in m(\Phi)^{i+1} P^{*}=m(\Phi)^{i} \tilde{P}$ if and only if for any short root $\alpha \in \Delta_{s}, m(\Phi)^{i+1} \mid m_{\alpha}(\lambda)$, and for any $\alpha \in \Delta_{l}, m(\Phi)^{i} \mid m_{\alpha}(\lambda)$. Suppose $e m(\Phi)^{j} \rho$ divides $\lambda$ with $e$ coprime to $m(\Phi)$; then $j \leq i$. Since $\lambda \in m(\Phi)^{i+1} P^{*}$, it follows that $\lambda$ is divisible by $\operatorname{em}(\Phi)^{j} \tilde{\rho}$, and hence the lcm can be taken with respect to such factors. This establishes the second case.

Remark 2.3. If we consider $\tilde{P}=m(\Phi) P^{*}$ as the weight lattice of the normalized dual root system with short roots having squared length 2 , then the dual weight lattice $\tilde{P}^{*}$ is just given by $P \supset \tilde{P}$. Hence the condition $\lambda \in m(\Phi)^{i+1} P^{*} \backslash m(\Phi)^{i+1} P$ for some $i \geq 0$ can be rewritten in the dual system to $\operatorname{read}$ as $\lambda \in m(\Phi)^{i} \tilde{P} \backslash m(\Phi)^{i+1} \tilde{P}^{*}$. It follows from the foregoing lemma for $\lambda \in m(\Phi)^{i} \tilde{P} \backslash m(\Phi)^{i+1} P$ that the definitions of both $D(\lambda)$ and $C(\lambda)$, considered as objects associated to the normalized dual root system, have exactly the same expression as when $\lambda \in m(\Phi)^{i} P \backslash m(\Phi)^{i+1} P^{*}$.

2.7. The main theorem. Our aim is to show that if $\lambda \in P^{++}$is neither a multiple of $\rho$ or of $\tilde{\rho}$, then $C(\lambda)$ is absolutely irreducible. However, the proof we have does not prove this in full generality and has a gap for a class of regular weights of $G_{2}$ and $F_{4}$. We make the following assumption (see Proposition 5.2):

Assumption NMFG: Consider the root systems given by $F_{4}$ and $G_{2}$, and let regular weights $\lambda \in P^{++}$be of the form

$$
\lambda=u \omega_{\alpha}+v \omega_{\beta}+d(\lambda) \rho, \quad d(\lambda)=(u, v),
$$

where $\omega_{\alpha}$ (resp. $\omega_{\beta}$ ) is the fundamental weight corresponding to the short (resp. long) corner root $\alpha$ (resp. $\beta$ ) in the Dynkin diagram such that the following inequalities are satsified:

$$
m(\Phi) v \geq u+d(\lambda) \text { and } u \geq v+d(\lambda) .
$$


Assumption $N M F G$ shows that for this class of weights, any $W$-invariant non-trivial factorization is non-monic (see Definition 5.2 for the definition of a factorization to be non-monic).

The main theorem of this paper is the following:

Theorem 2.3. With notation as above, let $\lambda \in P^{++}$be a dominant regular weight for the root system $\Phi$. Assume further that Assumption $N M F G$ is valid. If $\lambda \neq d \rho$ or $d \tilde{\rho}$ for some natural number $d$, then $C(\lambda)$ is absolutely irreducible, i.e., it is irreducible in the ring $\mathbb{C}[P]^{W}$.

Remark 2.4. By Remark 2.3, the use of duality reduces the proof of Theorem 2.3 to the case that

$$
\lambda \in m(\Phi)^{i} P \backslash m(\Phi)^{i+1} P^{*},
$$

for some $i \geq 0$. In this case, $D(\lambda)=S(d(\lambda) \rho)$ and we need to show that $C(\lambda)=$ $S(\lambda) / S(d(\lambda) \rho)$ is irreducible. We will achieve this by showing that if $C(\lambda)$ is reducible, then $\lambda \in m(\Phi)^{i+1} P^{*}$, contradicting our choice of $\lambda$ (see Section 5 ).

Remark 2.5. As for the case of $G L(r)$, it should be possible to obtain the irreducibility statement in the larger ring $\mathbb{C}[P]$. Analogous irreducibility results can be obtained for characters of rational representations of simple algebraic groups $G$. Here the irreducibility results hold in the bigger ring of regular functions of $G$, rather than the ring of regular invariant functions of $G$ (see Theorem 3.2).

Remark 2.6. The scaling operation $[d]: \mathbb{Z}[P] \rightarrow \mathbb{Z}[P]$ given by mulitplication by $d$ on $P$ allows a reformulation of the theorem using a slight modification of a ring used by Bourbaki $[\mathrm{B}$ ] in their formulation of the product expansion of the Weyl denominator. Let

$$
P_{\mathbb{Q}}=P \otimes_{\mathbb{Z}} \mathbb{Q},
$$

and denote by $\mathbb{C}\left[P_{\mathbb{Q}}\right]$ the group algebra of $P_{\mathbb{Q}}$ with coefficients in $\mathbb{C}$ (we use exponential notation). Observe that in this ring there exist elements which are infinitely factorizable, for example elements of the form $e^{p}-1$ for $p \in P$. Further an element $C \in \mathbb{C}[P]$ is irreducible as an element in $\mathbb{C}\left[P_{\mathbb{Q}}\right]$ if and only if $[d] C$ is irreducible in $\mathbb{C}[P]$ for all natural numbers $d$, where $[d]: \mathbb{C}[P] \rightarrow \mathbb{C}[P]$ denotes the algebra homomorphism induced by multiplication by $d$. Since $P$ and the dual weight lattice $P^{*}$ are commensurable in $P_{\mathbb{Q}}$, the two group rings $\mathbb{C}[P]$ and $\mathbb{C}\left[P^{*}\right]$ are both contained in $\mathbb{C}\left[P_{\mathbb{Q}}\right]$. In this ring of fractional Laurent polynomials, Theorem 2.3 can be reformulated to say that with the hypothesis of Theorem 2.3 , the element $C(\lambda)$ is irreducible in the ring $\mathbb{C}\left[P_{\mathbb{Q}}\right]^{W}$, where we can assume that $d(\lambda)=1$.

However, we do not work with this ring any further, as there is no clear advantage in working with this bigger ring.

2.8. A uniqueness property. The following theorem expresses a uniqueness property of 'generalized characters'; in particular, that the highest weight $\lambda$ can be recovered from knowing $C(\lambda)$ provided $C(\lambda)$ is non-trivial:

Theorem 2.4. Let $\mathcal{R}=(E, \Phi, \Delta)$ be a simple based root system and $\lambda_{i}, \mu_{i}, i=$ 1,2 , be dominant regular weights for $\mathcal{R}$. Assume that the weights $\mu_{1}, \mu_{2}$ are of generalized Weyl denominator type, i.e., they are an integral multiple of either $\rho$ or 
$\rho$. Assume further that $\mu_{i}$ divides $\lambda_{i}$ for $i=1,2$. Suppose there is an equality of the quotients,

$$
S\left(\lambda_{1}\right) / S\left(\mu_{1}\right)=S\left(\lambda_{2}\right) / S\left(\mu_{2}\right),
$$

and that these quotients are not equal to 1.

Then $\lambda_{1}=\lambda_{2}$ and $\mu_{1}=\mu_{2}$. In particular, if $\lambda_{1}$ is neither a multiple of $\rho$ or $\tilde{\rho}$, and $C\left(\lambda_{1}\right)=C\left(\lambda_{2}\right)$, then $\lambda_{1}=\lambda_{2}$.

The proof of the theorem is given in Section 9] it is by induction on the rank and uses properties of cofactor expansion. Together with Theorem 2.3 and the explicit factorizations of the generalized Schur-Weyl denominators, this gives a proof of the unique factorization of tensor products given by Theorem 1.1, but subject to Hypothesis $N M F G$. We refer to Section 9 for more details.

Remark 2.7. The uniqueness property is required for the proof of Proposition 5.2 that any symmetric factorization is non-monic, which in turn goes into the proof of Theorem 2.3.

\section{IRREDUCIBILITY PROPERTY FOR CHARACTERS OF IRREDUCIBLE REPRESENTATIONS OF SIMPLE ALGEBRAIC GROUPS}

In this section we extend the irreducibility results of the previous section to characters of finite dimensional representations of simple algebraic groups. For an algebraic group $H$, denote by $\mathcal{O}(H)$ the algebra of regular functions on $H$. If a group $L$ acts on $H$, we denote by $\mathcal{O}(H)^{L}$ the ring of regular functions on $H$ which are invariant with respect to the induced action of $L$ on $\mathcal{O}(H)$.

Let $G$ be a connected, simply connected, almost simple algebraic group over $\mathbb{C}$. Since $G$ is simply connected, by a theorem of Fossum, Iversen and Popov (see [FI, KKLV1, KKLV2]), the Picard group of $G$ is trivial. Hence the ring $\mathcal{O}(G)$ is factorial. We have the following:

Proposition 3.1. Let $G$ be a connected, simply connected, almost simple algebraic group over $\mathbb{C}$. Suppose $f \in \mathcal{O}(G)^{G}$ is an invariant regular function on $G$ with respect to the adjoint action of $G$ on itself. Then any irreducible factor of $f$ in $\mathcal{O}(G)$ is invariant with respect to the adjoint action of $G$ on itself.

In particular, if $f$ is an irreducible element in $\mathcal{O}(G)^{G}$, then it is irreducible in $\mathcal{O}(G)$.

Proof. Suppose there is a factorization

$$
f=p_{1} \cdots p_{r}
$$

in the ring $\mathcal{O}(G)$, where $p_{i}$ are irreducible elements in $\mathcal{O}(G)$. The group $G(\mathbb{C})$ acts by conjugation on $\mathcal{O}(G)$ leaving invariant the element $f$; hence it acts by permuting the irreducible factors (up to units) $p_{1}, \cdots, p_{r}$. Since $G(\mathbb{C})$ is connected, this implies that the permutation action is trivial,

$$
p_{i}^{g}=\xi_{i}(g) p_{i}, \quad i=1, \cdots, r,
$$

where $\xi_{i}(g)$ is a nowhere vanishing function on $G(\mathbb{C})$ and satisfies the 1-cocycle condition

$$
\xi_{i}(g h)=\xi_{i}(g)^{h} \xi_{i}(h) .
$$

From the regularity of the action of $G$ on $\mathcal{O}(G)$, we conclude that $\xi_{i}(g)$ is a regular function on $G$, hence a unit in $\mathcal{O}(G)$. By a theorem of Rosenlicht (KKLV1, page $78]$ ) the units in $\mathcal{O}[G]$ are just the constants. Thus the $G$ action is trivial on the 
units of $\mathcal{O}[G]$. This defines a homomorphism $g \mapsto \xi_{g}$ from the group $G(\mathcal{O})$ to $\mathbb{C}^{*}$. Since $G(\mathbb{C})$ has no abelian quotients, this implies the cocyle is trivial, and hence $p_{i}^{g}=p_{i}$ for any $g \in G(\mathbb{C})$. Hence the factorization given by equation (3.1) actually holds in $\mathcal{O}(G)^{G}$.

Let $T$ be a maximal torus in $G$. By Chevalley's restriction theorem, we have an isomorphism

$$
\mathcal{O}(G)^{G} \simeq \mathcal{O}(T)^{W}
$$

between the algebra $\mathcal{O}[G]^{G}$ and the algebra $\mathcal{O}(T)^{W}$ of Weyl group invariant functions of $H$. Let $X^{*}(T)$ denote the group of characters of $T$. The ring of regular functions $\mathcal{O}(T)$ on the torus $T$ can be identified with the group algebra $\mathbb{C}\left[X^{*}(T)\right]$. Since $G$ is simply connected, by a theorem of Chevalley it is known that these rings are isomorphic to the polynomial ring in $r$-variables, where $r$ is the dimension of $T$. The Lie algebra $\mathfrak{g}$ of $G$ is simple. Choosing a Borel subgroup $B \supset T$ of $G$ allows us to define simple roots, weights, etc. for $\mathfrak{g}$ too. The lattice of weights $P$ can be identified with the character group $X^{*}(T)$ of $T$. Hence we have an identification,

$$
\mathcal{O}(T)^{W} \simeq \mathbb{C}[P]^{W} .
$$

To each dominant, integral weight $\lambda$, denote the corresponding irreducible $G$ module with highest weight $\lambda$ by $V_{\lambda}$. Via the above isomorphism given by equation (3.2), the characters of the representation of $G$ and the Lie algebra on $V_{\lambda}$ can be identified. In particular, the irreducibility results of the previous section can be transferred to the context of invariant functions on the group. But we obtain a bit more by combining Theorem 2.3 and Proposition 3.1 (we have also incorporated the scaling operation):

Theorem 3.2. Let $G$ be a connected, simply connected, almost simple algebraic group over $\mathbb{C}$ of rank at least two. With respect to notation as above, let $\lambda$ be the highest weight of an irreducible representation of $G$. Suppose that $\lambda+\rho$ is not a multiple of either $\rho$ or $\tilde{\rho}$, and that $d(\lambda+\rho)=1$. Assume further that Assumption NMFG holds for the weight $\lambda+\rho$.

Then for any natural number $d$, the function $g \mapsto \chi_{\lambda}\left(g^{d}\right)$ is irreducible in the ring of regular functions of $G$.

Remark 3.1. For $G \simeq S L(r), r \geq 3$, this is the theorem mentioned in the abstract.

\section{Cofactor EXPANSIONS}

The proof of the unique decomposition of tensor products of irreducible representations of simple Lie algebras (see Theorem 1.1 given in $[R$ is by induction on the rank of the Lie algebra, by considering cofactor expansions of the Schur-Weyl elements occuring in the Weyl character formula. The same general principle is applied to the proof of the irreducibility property of characters, with the expectation that an inductive machinery can be set up.

4.1. Cofactor expansion for $G L(r)$. We first recall the cofactor expansion of the numerator of the Weyl character formula for $G L(r)$. Let $\lambda=\left(a_{1}>a_{2}>\cdots>\right.$ $\left.a_{r-1}>a_{r}=0\right)$ be a regular weight of $G L(r)$. The Schur-Weyl sum $S(\lambda)$ can be 
expressed as a determinant,

$$
\begin{aligned}
S(\lambda) & =\sum_{\sigma \in W} \epsilon(\sigma) x_{\sigma(1)}^{a_{1}} \cdots x_{\sigma(r)}^{a_{r}} \\
& =\operatorname{det}\left(x_{i}^{a_{j}}\right) .
\end{aligned}
$$

This admits a cofactor expansion,

$$
\begin{aligned}
S(\lambda)\left(x_{1}, \cdots, x_{r}\right) & =x_{1}^{a_{1}} S\left(\lambda^{(1)}\right)\left(x_{2}, \cdots, x_{r}\right)-x_{1}^{a_{2}} S\left(\lambda^{(2)}\right)\left(x_{2}, \cdots, x_{r}\right) \\
& +\cdots+(-1)^{r-2} x_{1}^{a_{r-2}} S\left(\lambda^{(r-2)}\right)\left(x_{2}, \cdots, x_{r}\right) \\
& +(-1)^{r-1}\left(x_{2} \cdots x_{r}\right)^{a_{r-1}} S\left(\lambda^{(r-1)}\right)\left(x_{2}, \cdots, x_{r}\right)
\end{aligned}
$$

where for $1 \leq i \leq r-2$,

$$
\lambda^{(i)}=\left(a_{1}, a_{2}, \cdots, a_{i-1}, a_{i+1}, \cdots, a_{r}\right)
$$

and

$$
\lambda^{(r-1)}=\left(a_{1}-a_{r-1}, \cdots, a_{r-2}-a_{r-1}, 0\right)
$$

are regular weights for $G L(r-1)$. For our purpose, we are interested only in the top (resp. bottom) two leading terms, and not the full cofactor expansion as such.

In terms of the fundamental weights defined as in Example 2.1, if

$$
\lambda=\sum_{i=1}^{r-1} m_{i}(\lambda) \omega_{i}
$$

with $m_{i}(\lambda)=a_{i}-a_{i+1}$, these weights can be expressed as

$$
\begin{aligned}
\lambda^{(1)} & =m_{2}(\lambda) \omega_{1}(r-1)+\cdots+m_{r-1}(\lambda) \omega_{r-2}(r-1), \\
\lambda^{(2)} & =\left(m_{1}(\lambda)+m_{2}(\lambda)\right) \omega_{1}(r-1)+\cdots+m_{r-1}(\lambda) \omega_{r-2}(r-1), \\
\lambda^{(r-1)} & =m_{1}(\lambda) \omega_{1}(r-1)+\cdots+m_{r-2}(\lambda) \omega_{r-2}(r-1),
\end{aligned}
$$

where we have put the argument $(r-1)$ to indicate that these are fundamental weights for $G L(r-1)$.

4.2. Cofactor expansion. Our aim is to generalize the foregoing cofactor expansion for $G L(r)$ to that of a general simple based root system $\mathcal{R}=(E, \Phi, \Delta)$ of rank $r$. The above interpretation of the cofactor expansion in terms of the fundamental weights leads us to consider corner roots in the Dynkin diagram of $\mathcal{R}$ and to decompose the weight lattice $P$ as a sum of the weight lattice corresponding to the simple Lie subalgebra corresponding to the corner root of corank one and the fundamental weight given by the corner root.

Choose a simple root $\alpha \in \Delta$. We will be primarily interested in the special case when $\alpha$ corresponds to a corner vertex in the Dynkin diagram of $\mathcal{R}$. Let $\Delta_{\alpha}=\Delta \backslash\{\alpha\}$, and let $\Phi_{\alpha} \subset \Phi$ be the subset of roots lying in the span of the roots generated by $\Delta_{\alpha}$. Let

$$
E_{\alpha}=\sum_{\alpha \in \Delta_{\alpha}} \mathbb{R} \alpha=\left\{\mu \in E \mid\left\langle\omega_{\alpha}^{*}, \mu\right\rangle=0\right\} .
$$

It is known that $\mathcal{R}_{\alpha}=\left(E_{\alpha}, \Phi_{\alpha}, \Delta_{\alpha}\right)$ is a based simple root system of rank $r-1$.

Let $W_{\alpha}$ denote the Weyl group of $\mathcal{R}_{\alpha}$. It can be identified with the subgroup of $W$ generated by the fundamental reflections $s_{\beta}$ for $\beta \in \Delta_{\alpha}$. The following lemma provides a $W_{\alpha}$-equivariant decomposition of $P$, a complement to $E_{\alpha}$ inside $E$ (see [R, Lemma 3]). 
Lemma 4.1. Let $\omega_{\alpha}$ (resp. $\omega_{\alpha}^{*}$ ) denote the fundamental weight (resp. coweight) corresponding to the simple root $\alpha$. The isostropy group of $\omega_{\alpha}^{*}$ is precisely $W_{\alpha}$. There is $W_{\alpha}$-equivariant decomposition,

$$
E=\mathbb{R} \omega_{\alpha} \oplus E_{\alpha} \quad \text { and } \quad E^{*}=\mathbb{R} \omega_{\alpha}^{*} \oplus E_{\alpha}^{*} .
$$

Via the above decomposition, the rational weight lattice $P \otimes \mathbb{Q}$ admits a $W_{\alpha^{-}}$ equivariant splitting,

$$
P \otimes \mathbb{Q}=\mathbb{Q} \omega_{\alpha} \oplus P_{\alpha} \otimes \mathbb{Q},
$$

where the weight lattice $P_{\alpha}$ of the root system $\Phi_{\alpha}$ can be identified with the subspace of $P$,

$$
P_{\alpha}=\operatorname{Ker}\left(\omega_{\alpha}^{*}\right)=\left\{\pi \in P \mid\left\langle\omega_{\alpha}^{*}, \pi\right\rangle=0\right\} .
$$

Further, there is a $W_{\alpha}$-equivariant inclusion,

$$
P \subset \mathbb{Z} \frac{\omega_{\alpha}}{\left\langle\omega_{\alpha}^{*}, \omega_{\alpha}\right\rangle} \oplus P_{\alpha}
$$

The last assertion follows from the fact if $\mu \in P$ takes integral values on the simple coroots, then its projection to $E_{\alpha}$ also takes integral values on the simple coroots $\beta^{*}, \beta \in \Delta_{\alpha}$, as $\omega_{\alpha}$ is orthogonal to all such $\beta^{*}$.

Denote by $l_{\alpha}$ the rational weight,

$$
l_{\alpha}=\frac{1}{\left\langle\omega_{\alpha}^{*}, \omega_{\alpha}\right\rangle} \omega_{\alpha}
$$

With respect to the decomposition, a weight $\pi \in P \otimes \mathbb{Q}$ can be written as

$$
\pi=\omega_{\alpha}^{*}(\pi) l_{\alpha}+\pi^{\alpha},
$$

where $\pi^{\alpha} \in P_{\alpha, 0}=P_{\alpha} \otimes \mathbb{Q}$, defined by the above equation, is the $W_{\alpha}$-equivariant projection of $\pi$ along $\omega_{\alpha}$ to $E_{\alpha}$. The integer $\omega_{\alpha}^{*}(\pi)$ (or the rational number $\omega_{\alpha}^{*}(\pi) / \omega_{\alpha}^{*}\left(\omega_{\alpha}\right)$ ) will be referred to as the degree of $\pi$ along $l_{\alpha}$ (or along $\omega_{\alpha}$ or $\alpha)$.

Let $\omega$ be a fundamental weight of $\Phi$ distinct from $\omega_{\alpha}$. It follows from equation (4.8) that $\omega^{\alpha}$ is a fundamental weight for the root system $\Phi_{\alpha}$. In particular, the projection $\rho^{\alpha}$ of $\rho=\rho(\Phi)$ is the sum of the fundamental weights of $\Phi_{\alpha}$. i.e., equal to the Weyl weight of the root system $\Phi_{\alpha}$.

Suppose $U$ is an element of $\mathbb{C}[P]$. Write

$$
U=\sum_{\mu \in P} a_{\mu}(U) e^{\mu}
$$

Define $P(U)$ to be the finite set of weights occurring in $U$,

$$
P(U)=\left\{\mu \in P \mid a_{\mu}(U) \neq 0\right\} .
$$

Expand $U$ in terms of the 'degree along $\alpha$ ' as

$$
U=\sum_{i \geq 0} U_{\alpha, u-i}^{\prime}
$$

where

$$
U_{\alpha, u-i}^{\prime}=\sum_{\mu \in P(U), \omega_{\alpha}^{*}(\mu)=u-i} a_{\mu}(U) e^{\mu} .
$$

If $U$ is $W$-invariant, then the terms $U_{\alpha, u-i}^{\prime}$ are $W_{\alpha}$-invariant. The term $U_{\alpha, u-i}^{\prime}$ can also be written as

$$
U_{\alpha, u-i}^{\prime}=e^{(u-i) l_{\alpha}} U_{\alpha, u-i}
$$


where we now consider $U_{\alpha, i}$ as an element of $\mathbb{C}\left[P_{\alpha}\right]$. Define the cofactor expansion of $U$ along $\alpha$ as

$$
U=\sum_{i \geq 0} e^{(u-i) l_{\alpha}} U_{\alpha, u-i}
$$

The element $U_{\alpha, u} \in \mathbb{C}\left[P_{\alpha}\right]$ will also be referred to as the leading coefficient of $U$ along $\alpha$.

For any integer $i \geq 0$, we refer to the term $U_{\alpha, u-i}$ as the $i$-th codegree term in the cofactor expansion of $U$ along $\alpha$ (see Section 9 where we use this notation).

Example 4.1. If we consider the cofactor expansion of $S(\lambda)$ for $G L(r)$, the top degree coefficient is the leading coefficient corresponding to the cofactor expansion along the simple corner root $e_{1}-e_{2}$ (standard notation). Up to a monomial term, the constant term is the leading coefficient in the cofactor expansion of $S(\lambda)$ along the other corner fundamental root $e_{r-1}-e_{r}$.

4.3. Cofactor expansion of $S(\lambda)$. We now describe the cofactor expansion of the Schur-Weyl sum $S(\lambda)$. Let $W(\alpha)$ be a set of right coset representatives for $W_{\alpha}$ in $W$, i.e., a section for the projection map $W \rightarrow W_{\alpha} \backslash W$. For any element $w \in W_{\alpha}$ and $s \in W$, the value

$$
\omega_{\alpha}^{*}(w s \lambda)=\left(w^{-1} \omega_{\alpha}^{*}\right)(s \lambda)=\omega_{\alpha}^{*}(s \lambda)
$$

is a constant for any weight $\lambda$. Hence the Schur-Weyl sum $S(\lambda)$ can be expanded as

$$
\begin{aligned}
S(\lambda) & =\sum_{d \in \mathbb{Z}} e^{d l_{\alpha}}\left(\sum_{w \in W_{d}} \epsilon(w) e^{(w \lambda)^{\alpha}}\right) \\
& =\sum_{s \in W(\alpha)} e^{\omega_{\alpha}^{*}(s \lambda) l_{\alpha}} S\left((s \lambda)^{\alpha}\right),
\end{aligned}
$$

where $W_{d}=\left\{w \in W \mid \omega_{\alpha}^{*}(w \lambda)=d\right\}$,

and for each $s \in W(\alpha), S\left((s \lambda)^{\alpha}\right)$ refers to the Schur-Weyl sum of the weight $(s \lambda)^{\alpha}$ belonging to the root system $\mathcal{R}_{\alpha}$. In the above notation,

$$
S(\lambda)_{\alpha, d}=\sum_{s} S\left((s \lambda)^{\alpha}\right)
$$

where the sum ranges over $s \in W(\alpha)$ such that $\omega_{\alpha}^{*}(s \lambda)=d$.

We are interested in the first two leading terms in the above expansion. Given a regular weight $\lambda \in P_{+}$, define

$$
\begin{aligned}
& a_{\alpha, 1}(\lambda)=\max \left\{w \lambda\left(\omega_{\alpha}^{*}\right) \mid w \in W\right\}, \\
& a_{\alpha, 2}(\lambda)=\max \left\{w \lambda\left(\omega_{\alpha}^{*}\right) \mid w \in W \text { and } w \lambda\left(\omega_{\alpha}^{*}\right) \neq a_{\alpha, 1}(\lambda)\right\} .
\end{aligned}
$$

The following lemma is proved in $[\underline{\mathrm{R}}$, Lemma 4]:

Lemma 4.2. Let $\lambda$ be a regular weight in $P_{+}$and $\alpha \in \Delta$.

(1) The largest value $a_{\alpha, 1}(\lambda)$ of $(w \lambda)\left(\omega_{\alpha}^{*}\right)$ for $w \in W$ is attained precisely for $w \in W_{\alpha}$. In particular,

$$
a_{\alpha, 1}(\lambda)=\left\langle\omega_{\alpha}^{*}, \lambda\right\rangle
$$


(2) The second highest value of $a_{\alpha, 2}(\lambda)$ is attained precisely for $w$ in $W_{\alpha} s_{\alpha}$, and the value is given by

$$
a_{\alpha, 2}(\lambda)=\omega_{\alpha}^{*}\left(s_{\alpha} \lambda\right)=a_{\alpha, 1}(\lambda)-\left\langle\alpha^{*}, \lambda\right\rangle=a_{\alpha, 1}(\lambda)-m_{\alpha}(\lambda) .
$$

Assume now that $\alpha$ is a corner root. Then $\mathcal{R}_{\alpha}$ is simple. As a corollary of the above discussion, we obtain the first two terms for the cofactor expansion of $S(\lambda)$ along $\omega_{\alpha}$ :

Lemma 4.3. With notation as above, let $\lambda=\sum_{\beta \in \Delta} m_{\beta}(\lambda) \omega_{\beta}$. The cofactor expansion of $S(\lambda)$ given by equation (4.10) is

$$
S(\lambda)=e^{a_{\alpha, 1}(\lambda) l_{\alpha}} S\left(\lambda^{\alpha}\right)-e^{a_{\alpha, 2}(\lambda) l_{\alpha}} S\left(\left(s_{\alpha} \lambda\right)^{\alpha}\right)+L(\lambda),
$$

where $L(\lambda)$ denotes the terms of degree along $l_{\alpha}$ less than the second highest degree. In terms of fundamental weights,

$$
\begin{aligned}
\lambda^{\alpha} & =\sum_{\beta \in \Delta_{\alpha}} m_{\beta}(\lambda) \omega_{\beta}^{\alpha}, \\
\left(s_{\alpha} \lambda\right)^{\alpha} & =\lambda^{\alpha}+\left|m_{\alpha_{n}}(\alpha)\right| m_{\alpha}(\lambda) \omega_{\alpha_{n}}^{\alpha},
\end{aligned}
$$

where $\alpha_{n}$ is the unique root connected to $\alpha$ in the Dynkin diagram of $\mathcal{R}$.

Proof. We need to prove only the last formula. By definition,

$$
s_{\alpha} \lambda=\lambda-\left\langle\alpha^{*}, \lambda\right\rangle \alpha=\lambda-m_{\alpha}(\lambda) \alpha .
$$

Since $\mathcal{R}$ is simple and $\alpha$ is a corner root, the term $m_{\beta}(\alpha)=\left\langle\beta^{*}, \alpha\right\rangle$ vanishes if $\beta$ is different from $\alpha$ and $\alpha_{n}$. Hence, in terms of fundamental weights,

$$
\alpha=\sum_{\beta \in \Delta} m_{\beta}(\alpha) \omega_{\beta}=2 \omega_{\alpha}+m_{\alpha_{n}}(\alpha) \omega_{\alpha_{n}},
$$

where $m_{\alpha_{n}}(\alpha)=\left\langle\alpha_{n}^{*}, \alpha\right\rangle$ is a negative integer. Putting all this together yields

$$
\begin{aligned}
\left(s_{\alpha} \lambda\right)^{\alpha} & =\lambda^{\alpha}+\left|m_{\alpha_{n}}(\alpha)\right| m_{\alpha}(\lambda) \omega_{\alpha_{n}}^{\alpha} \\
& =\left(m_{\alpha_{n}}(\lambda)+\left|m_{\alpha_{n}}(\alpha)\right| m_{\alpha}(\lambda)\right) \omega_{\alpha_{n}}^{\alpha}+\sum_{\beta \neq \alpha, \alpha_{n}} m_{\beta}(\lambda) \omega_{\beta}^{\alpha} .
\end{aligned}
$$

Remark 4.1. Since $m_{\alpha_{n}}(\alpha)=\left\langle\alpha_{n}^{*}, \alpha\right\rangle$ and $\alpha_{n}^{*}=2 \alpha_{n} /\left\langle\alpha_{n}, \alpha_{n}\right\rangle$, it can be seen that

$$
\left|m_{\alpha_{n}}(\alpha)\right|= \begin{cases}m(\Phi) & \text { if } \alpha \text { is long and } \alpha_{n} \text { is short } \\ 1 & \text { otherwise. }\end{cases}
$$

Example 4.2. For $G L(r),\left|m_{\alpha_{n}}(\alpha)\right|=1$, and this gives the formula expressed in equation (4.2):

$$
\lambda^{(2)}=m_{1}(\lambda) \omega_{1}(r-1)+\lambda^{(1)} .
$$

4.4. Eisenstein criterion. We now state the equivalent in our context of the observation used in the proof of the classical Eisenstein criterion regarding irreducibility of polynomials.

Lemma 4.4 (Eisenstein criterion). Assume that Theorem 2.3 holds for any irreducible based root system of rank less than that of $\mathcal{R}=(E, \Phi, \Delta)$. Let $\lambda$ be a dominant regular weight and $U$ be a factor of $S(\lambda)$. Then for any $\alpha \in \Delta$ and for any $i<m_{\alpha}(\lambda)$, the leading term $U_{\alpha, u}$ in the cofactor expansion given by equation (4.9) divides $U_{\alpha, u-i}$ in the ring $\mathbb{C}\left[P_{\alpha}\right]$. 
Proof. Let $\mu=\lambda^{\alpha}$. By Corollary 9.2, $S(\mu)$ is a seperable element in $\mathbb{C}\left[P_{\alpha}\right]$. Further, the terms of degree $d$ in the cofactor expansion of $S(\lambda)$ along $\alpha$ vanish in the range

$$
a_{\alpha, 1}(\lambda)>d>a_{\alpha, 2}(\lambda)=a_{\alpha, 1}(\lambda)-m_{\alpha}(\lambda) .
$$

The proof of the classical Eisenstein criterion now applies to establish the lemma.

\section{Key Lemmas AND the PROOF OF the MAIN THEOREM}

In this section we present the key propositions and the deduction of the main theorem from these propositions. We present this for a general simple Lie algebra. The proof for $G L(r)$ is exactly the same, and we state the case for $G L(r)$ only when it is convenient to do so.

5.1. An arithmetical preliminary. We first do a preliminary reduction and show that any putative factorization can be considered over suitable 'arithmetical' rings: let $N$ be a natural number (to be chosen later, depending on the dominant regular $\lambda$ ), and let $\mathcal{O}$ be a ring of characteristic zero satisfying the following property $\mathbf{A}_{N}$ :

(1) The ring $\mathcal{O}$ has class number one.

(2) The prime numbers $p \leq N$ are not units in $\mathcal{O}$.

(3) $\mathcal{O}$ contains the roots of unity $\zeta_{k}$ of order $k \leq N$.

Lemma 5.1. Suppose there is a factorization in $\mathbb{C}[P]^{W}$ (or in $\mathbb{C}[P]$ ),

$$
C(\lambda, d)=Q R \text {. }
$$

Given any natural number $N$, there exists a UFD $\mathcal{O} \subset \mathbb{C}$ satisfying property $\mathbf{A}_{N}$ such that $Q$ and $R$ are in $\mathcal{O}_{N}[P]^{W}$ (resp. $\left.\mathcal{O}_{N}[P]\right)$ up to multiplying by constants.

Proof. The lemma goes under the name of Lefschetz principle, and for the sake of completeness we give a proof based on Gauss' lemma: Suppose $A$ is a UFD, and $C \in A\left[x_{1}, \cdots, x_{n}\right]$ is a polynomial such that the gcd of its coefficients is a unit in $A$. Then if $C$ admits a factorization in $K\left[x_{1}, \cdots, x_{n}\right]$ where $K$ is the quotient field of $A$, then it admits a factorization in $A\left[x_{1}, \cdots, x_{n}\right]$.

To prove the lemma, by attaching the coefficients of $Q$ and $R$, we can assume that the factorization is over a finitely generated field $E$ over $\mathbb{Q}$. Let $F$ be the algebraic closure of $\mathbb{Q}$ in $E$; this is a finite extension of $\mathbb{Q}$, and $E$ is a finitely generated purely transcendental extension over $F$. We can write $E$ as the quotient field of $A=F\left[\xi_{1}, \cdots, \xi_{k}\right]$ for some algebraically independent generators $\xi_{1}, \cdots, \xi_{k}$. By Gauss' lemma, we can assume (after multiplying by some units) that the factors $Q$ and $R$ belong to $A\left[x_{1}, \cdots, x_{n}\right]$. By considering them as polynomials in the variables $\xi_{1}, \cdots, \xi_{k}, x_{1}, \cdots, x_{n}$ and by degree considerations, we see that the coefficients of $Q$ and $R$ have to lie in the number field $F$, which we can assume contains the $N$-th roots of unity.

Let $\mathcal{O}_{F}$ be the ring of algebraic integers in $F$. By inverting the primes $q \geq N$, we get a semilocal ring $\mathcal{O}_{N}$. This is a UFD and the primes $p<N$ are not units. By Gauss' lemma, the factorization is defined over $\mathcal{O}_{N}[P]^{W}$ (resp. $\mathcal{O}_{N}[P]$ by using a version of Gauss' lemma over Laurent rings), and this proves the lemma. 
5.2. Key proposition. The heart of the proof of the main theorem is the following proposition:

Proposition 5.1. Let $\mathcal{R}=(E, \Phi, \Delta)$ be a simple based root datum of rank l. Let $\mu$ be a regular weight in $P_{++}$and $\eta=\mu+c \omega_{\alpha}$ for some positive integer $c$, where $\omega_{\alpha}$ is the fundamental weight corresponding to $\alpha \in \Delta$. Assume that $\alpha$ is a corner root in the Dynkin diagram for $\mathcal{R}$ if $\mathcal{R}$ is not simply laced. Assume further that Theorem 2.3 is valid for all simple Lie algebras of rank less than $l$.

Let $d, e$ be natural numbers with $d$ dividing $d(\mu)$, e and $c$, and e dividing $d(\mu)$. Let $N$ be a natural number greater than

$$
N(\eta):=2 m(\Phi)\left(\sum_{\alpha \in \Delta} m_{\alpha}(\eta)\right),
$$

and let $\mathcal{O}$ be as in Lemma 5.1. Suppose there exist symmetric elements $U, V \in$ $\mathcal{O}_{N}[P]^{W}$ which are not units in $K_{N}[P]^{W}$, where $K_{N}$ is the quotient field of $\mathcal{O}_{N}$, and which satisfy the following:

$$
U V=C(\mu, d)
$$

- The factor $V$ of $C(\mu, d)$ divides $C(e \rho, d)$.

- There exist elements $X, Y \in \mathcal{O}[P]$ such that

$$
U X+V Y=C(\eta, d) \text {. }
$$

Then the following hold:

(1) If $\mathcal{R}$ is simply laced or if $\alpha$ is a short root, then $(e, c)>d$.

(2) If $\mathcal{R}$ is not simply laced and $\alpha$ is a long root, then $(e, m(\Phi) c)>d$.

Remark 5.1. The proof of Theorem 2.3 is by induction on the rank $r$, where the above proposition will be used for root systems of rank $l<r$. Thus for the proof of the main theorem, we will not require the $F_{4}$ and the $G_{2}$ cases of the foregoing proposition.

Remark 5.2. When $\Phi$ is of rank one, the above proposition translates to a statement about factors of a cyclotomic polynomial, such that their combination is equal to another cyclotomic polynomial. The proof of the proposition is arithmetic and is given in Section 6. Further, it is not required that the factors $U$ and $V$ be invariant.

For higher ranks, the arithmetical proof for $s l(2)$ does not generalize, as the arithmetical properties of a character, if any, are not easy to understand. The proof is by induction on the rank and is given in Section 7 The invariant condition on the factors is required to ensure that there is a corner root such that the leading coefficients of $U$ and $V$ are not units (see Proposition 5.2).

5.3. Non-monic invariant factorizations. In order to reduce the proof of the main theorem to that of Proposition 5.1. we need to rule out certain types of factorizations: factorizations such that in the cofactor expansion along any corner root, at least one of the factors is monic. In the case of $G L(r)$ we are working with symmetric homogeneous polynomials in $r$-variables. We look at the polynomials as polynomials in $x_{1}$ with coefficient polynomials in the variables $x_{2}, \cdots, x_{r}$. In this case, we want to rule out factorizations, where one factor is monic and the constant coeffecient of the other factor is monomial.

Let $\mathcal{R}=(E, \Phi, \Delta)$ be a based simple root system of rank $r$. Let $\alpha \in \Delta$ be a corner root in the Dynkin associated to $E$. 
Definition 5.1. An element $U \in \mathbb{C}[P]$ is said to be monic with respect to $\alpha$ if there is an unique weight $\mu_{\alpha} \in P(U)$ with maximum degree amongst all the weights occurring in $U$, i.e.,

$$
\omega_{\alpha}^{*}\left(\mu_{\alpha}\right) \geq \omega_{\alpha}^{*}(\mu) \quad \forall \mu \in P(U),
$$

with equality if and only if $\mu=\mu_{\alpha}$.

If we further assume that $U \in \mathbb{C}[P]^{W}$, since the ring $\mathbb{C}[P]^{W}$ is isomorphic to a polynomial ring in $r$-variables, the element $\mu_{\alpha}$ is well defined. By symmetry we observe that $\mu_{\alpha}$ is fixed by the subgroup $W_{\alpha}$ of the Weyl group $W$ fixing $\omega_{\alpha}$. Hence $\mu_{\alpha}=u \omega_{\alpha}$ for some integer $u$.

Definition 5.2. Let $C$ be an element in $\mathbb{C}[P]$, and suppose there is a factorization $C=U V$ in $\mathbb{C}[P]$. The factorization is said to be non-monic if there exists a corner root $\alpha$ in the Dynkin diagram of $\mathcal{R}$ such that both $U$ and $V$ are not monic along $\alpha$.

We show that any possible invariant factorization is non-monic under Assumption $N M F G$ :

Proposition 5.2. Let $\mathcal{R}=(E, \Phi, \Delta)$ be a simple based root system of rank $r$. Assume that Theorem 2.3 is valid for all simple root systems of rank strictly less than $r$. Let $\lambda$ be a dominant regular weight for $(E, \Phi, \Delta)$, belonging to $m(\Phi)^{i} P \backslash m(\Phi)^{i+1} P^{*}$ for some $i \geq 0$. Suppose there is a factorization

$$
C(\lambda)=U V
$$

where both $U$ and $V$ are in $\mathbb{C}[P]^{W}$. Then this factorization is non-monic, except when the root system is of type either $G_{2}$ or $F_{4}$ and the weight $\lambda$ is of the form

$$
\lambda=u \omega_{\alpha}+v \omega_{\beta}+d(\lambda) \rho, \quad d(\lambda)=(u, v),
$$

where $\omega_{\alpha}$ (resp. $\omega_{\beta}$ ) is the fundamental weight corresponding to the short (resp. long) corner root $\alpha$ (resp. $\beta$ ) in the Dynkin diagram such that the following inequalities are satsified:

$$
m(\Phi) v \geq u+d(\lambda) \quad \text { and } \quad u \geq v+d(\lambda) .
$$

Remark 5.3. Proposition 5.2 can also be considered as establishing the irreducibility property in $\mathbb{C}[P]^{W}$ for a "general" weight $\lambda$, i.e., those for which the leading coeffecient of $C\left(\lambda^{\alpha}, d(\lambda)\right)$ along any corner root $\alpha$ is irreducible.

The proof of Proposition 5.2 is essentially based on the use of Eisenstein criterion (see Lemma 4.4), to obtain lower bounds for the degrees along the corner roots of the factors $U$ and $V$. These bounds suffice except for a class of weights for $F_{4}$ and $G_{2}$ (Assumption $N M F G$ ). In order to apply the Eisenstein criteria, we require that the leading coefficients in the cofactor expansions of $S(\lambda)$ along any corner root be separable. The leading coefficients are again of the form $S(\mu)$, but for smaller rank simple root systems. The separability is given by Corollary 9.2

To obtain the lower bounds, we also require a coprimality result given by Corollary 9.4 stating that the first two leading coefficients of $S(\lambda)$ upon dividing by $S(d(\lambda) \rho)$ are coprime. These results together with Theorem 2.4 establishing a uniqueness property of $C(\lambda)$ will be proved in Section 9. It is for proving these results that we assume the validity of the main Theorem 2.3. for all simple root systems of rank strictly less than $r$. 
5.4. Proof of the main theorem. We now prove Theorem 2.3 assuming the validity of Propositions 5.1 and 5.2. First of all, by duality (see Remark 2.4) we can assume that $\lambda$ is an element of $m(\Phi)^{i} P \backslash m(\Phi)^{i+1} P^{*}$ for some $i \geq 0$. In this case, the greatest common divisor $D(\lambda)$ of the 'obvious' Weyl denominator type factors of $S(\lambda)$ is $S(d(\lambda) \rho)$, and we need to show that $C(\lambda, d)=S(\lambda) / S(d(\lambda) \rho)$ is irreducible, where $d=d(\lambda)$.

Suppose there is a factorization in $\mathbb{C}[P]^{W}$ (or in $\mathbb{C}[P]$ ),

$$
C(\lambda, d)=Q R \text {. }
$$

Choose a ring $\mathcal{O}$ as in Proposition [5.1, with

$$
N \geq N(\lambda)=2 m(\Phi)\left(\sum_{\alpha \in \Delta} m_{\alpha}(\lambda)\right) .
$$

By Proposition 5.2, choose a corner root $\alpha_{0}$ of the Dynkin diagram of $\mathcal{R}$ such that the leading coefficients $U$ (resp. $V$ ) of $Q$ (resp. $R$ ) along $\alpha_{0}$ with respect to the co-factor expansion of $Q$ and $R$ along $\alpha_{0}$ are not units in the $\operatorname{ring}(\mathcal{O} \otimes \mathbb{Q})\left[P_{\alpha_{0}}\right]^{W_{\alpha_{0}}}$. Let

$$
\mu=\lambda^{\alpha_{0}} .
$$

Then

$$
C(\mu, d(\lambda) \rho)=U V .
$$

The main observation that in conjunction with Proposition 5.1 will allow us to prove Theorem 2.3 is the following lemma:

Lemma 5.2. With the above notation, suppose $\lambda$ is an element of $m(\Phi)^{i} P \backslash$ $m(\Phi)^{i+1} P^{*}$ for some $i \geq 0$. Then

$$
d=d(\lambda)=\left(d\left(\lambda^{\alpha_{0}}\right), d\left(\left(s_{\alpha_{0}} \lambda\right)^{\alpha_{0}}\right)\right) .
$$

Proof. From equation (4.12) occurring in Lemma 4.3

$$
\left(s_{\alpha_{0}} \lambda\right)^{\alpha_{0}}=\lambda^{\alpha_{0}}+\left|m_{\alpha}\left(\alpha_{0}\right)\right| m_{\alpha_{0}}(\lambda) \omega_{\alpha}^{\alpha_{0}} .
$$

If $\left|m_{\alpha}\left(\alpha_{0}\right)\right|=1$, then the lemma follows easily. By equation (4.16), if $\left|m_{\alpha}\left(\alpha_{0}\right)\right| \neq 1$, then it is equal to $m(\Phi)$, the corner root $\alpha_{0}$ is long and the neighbouring fundamental root $\alpha$ in the Dynkin diagram of $\mathcal{R}$ is short. We have to consider the exact exponent of $m(\Phi)$ that divides the concerned quantities. By assumption, $m(\Phi)^{i} \| d$, as there is a short root $\gamma \in \Delta$ for which $m(\Phi)^{i} \| m_{\gamma}(\lambda)$. If $\gamma \neq \alpha$, then the lemma follows. If $\gamma=\alpha$, from the fact that $m(\Phi)^{i} \mid m_{\alpha_{0}}(\lambda)$ it follows that the exponent to which $m(\Phi)$ divides

$$
m_{\alpha}\left(\left(s_{\alpha_{0}} \lambda\right)^{\alpha_{0}}\right)=m_{\alpha}(\lambda)+m(\Phi) m_{\alpha_{0}}(\lambda)
$$

is exactly $i$. Hence the exponent to which $m(\Phi)$ divides $\left(d\left(\lambda^{\alpha_{0}}\right), d\left(\left(s_{\alpha_{0}} \lambda\right)^{\alpha_{0}}\right)\right)$ is exactly $i$. This proves the lemma.

The cofactor expansion of $Q$ and $R$ is given by

$$
Q=\sum_{i \geq 0} e^{(q-i) l_{\alpha_{0}}} Q_{\alpha_{0}, q-i}, \quad R=\sum_{i \geq 0} e^{(r-i) l_{\alpha_{0}}} R_{\alpha_{0}, r-i},
$$

where we have denoted by $q$ (resp. $r$ ) the degrees of the cofactor expansions of $Q$ and $R$ along $\alpha$. Further, since $Q$ and $R$ are assumed to be invariant, the coefficients $Q_{\alpha_{0}, q-i}$ and $R_{\alpha_{0}, r-i}$ are $W_{\alpha_{0}}$-invariant. 
By the Eisenstein criterion Lemma 4.4, the leading coefficient $U$ (resp. $V$ ) of $Q$ (resp. $R$ ) along $\alpha_{0}$ divides $Q_{\alpha_{0}, q-i}$ (resp. $R_{\alpha_{0}, r-i}$ ) for $i<m_{\alpha_{0}}(\lambda)$. Upon substituting the cofactor expansions of $Q$ and $R$ in equation (5.3), we see that there exist elements $X, Y$ in the ring $\mathcal{O}\left[P_{\alpha_{0}}\right]^{W_{\alpha_{0}}}$ such that

$$
U X+V Y=C(\eta, d(\lambda)) .
$$

Here $\eta$ is given by (4.13) in Lemma 4.3 .

$$
\eta=\left(s_{\alpha_{0}} \lambda\right)^{\alpha_{0}}=\mu+c \omega_{\alpha}^{\alpha_{0}},
$$

where $\alpha$ is the unique root in the Dynkin diagram attached to $\mathcal{R}$ that is connected to the corner root $\alpha_{0}$, and $c$ is given by equation (4.16)

$$
\begin{aligned}
c & =m_{\alpha_{0}}(\lambda)\left|m_{\alpha}\left(\alpha_{0}\right)\right| \\
& = \begin{cases}m(\Phi) m_{\alpha_{0}}(\lambda) & \text { if } \alpha_{0} \text { is long and } \alpha \text { is short, } \\
m_{\alpha_{0}}(\lambda) & \text { otherwise. }\end{cases}
\end{aligned}
$$

From these equations, it follows in particular that

$$
N \geq N(\lambda) \geq N(\eta)
$$

To see this, the non-trivial case is when $\alpha_{0}$ is long and $\alpha$ is short; but from the classification of simple Lie algebras, the root system $\mathcal{R}^{\alpha_{0}}$ is simply laced. Hence the above inequality follows.

We now analyze the possible factorizations of $C(\mu, d(\lambda))$ assuming the validity of Theorem 2.3 for $\mathcal{R}_{\alpha_{0}}$, and apply Proposition 5.1 to the root system $\mathcal{R}_{\alpha_{0}}$ together with equation (5.6) to arrive at a contraduction (and thus prove Theorem 2.3).

Case (i). We first analyze the case where the root system $\mathcal{R}_{\alpha_{0}}$ is either simply laced, or if not simply laced, then $\mu=\lambda^{\alpha_{0}}$ does not belong to $m\left(\Phi_{\alpha_{0}}\right)^{i+1} P_{\alpha_{0}}^{*}$.

If $\mu$ is not of Weyl denominator type, then

$$
C(\mu, d(\lambda))=C(\mu, d(\mu)) C(d(\mu), d(\lambda)) .
$$

Here by the inductive hypothesis $C(\mu, d(\mu))$ is absolutely irreducible by Theorem 2.3. In this case, let

$$
e=d(\mu), \quad d=d(\lambda), \quad \text { and } \quad d \mid e .
$$

We can assume by switching $U$ and $V$ if required, that $V$ divides $C(e \rho, d)$.

If $\mu=e \rho$ is of Weyl denominator type, then $V$ (and $U$ too) divides $C(e \rho, d)$.

Now suppose that we are not in the case where $\alpha_{0}$ is long and $\alpha$ is a short root. Then, by equation (5.10),

$$
c=m_{\alpha_{0}}(\lambda)
$$

Applying Proposition 5.1

$$
d=d(\lambda)=\left(d(\mu), m_{\alpha_{0}}(\lambda)\right)>d,
$$

and this contradicts Lemma 5.2

Assume now that we are in the situation where $\alpha_{0}$ is long and $\alpha$ is short. In this case, the root system $\mathcal{R}^{\alpha_{0}}$ is simply laced and let $e=d(\mu), c=m(\Phi) m_{\alpha_{0}}(\lambda)$. By Proposition 5.1 .

$$
\left(d(\mu), m(\Phi) m_{\alpha_{0}}(\lambda)\right)>d .
$$


We concentrate only on the contribution by the prime number $m(\Phi)$ to the computation of the gcd's. Since we have assumed that $\lambda \in m(\Phi)^{i} P \backslash m(\Phi)^{i+1} P^{*}$, this implies

$$
d(\mu)=m(\Phi)^{i} d^{\prime}(\mu) \quad \text { with } \quad\left(d^{\prime}(\mu), m(\Phi)\right)=1 .
$$

Write $d=m(\Phi)^{i} d^{\prime}$ with $d^{\prime}$ coprime to $m(\Phi)$. Let $m_{\alpha_{0}}(\lambda)=m(\Phi)^{i} m_{\alpha_{0}}^{\prime}(\lambda)$, where $m_{\alpha_{0}}^{\prime}(\lambda)$ is a natural number. Substituting, we get

$$
\left(d^{\prime}(\mu) m(\Phi)^{i}, m(\Phi)^{i+1} m_{\alpha_{0}}^{\prime}(\lambda)\right)>m(\Phi)^{i} d^{\prime} .
$$

On the other hand, Lemma 5.2 yields

$$
\left(d^{\prime}(\mu), m_{\alpha_{0}}(\lambda)\right)=d^{\prime} .
$$

These equations imply that $m(\Phi)$ divides $d^{\prime}(\mu)$, contradicting the choice of $\lambda$.

Case (ii). Now suppose that the root system $\mathcal{R}_{\alpha_{0}}$ is not simply laced and $\mu \in m\left(\Phi_{\alpha_{0}}\right)^{i+1} P_{\alpha_{0}}^{*}$. From the classification of the Dynkin diagrams, this can happen only when both $\alpha_{0}$ and its neighbour $\alpha \in \Delta$ have the same lengths. Further, $m(\Phi)=m\left(\Phi_{\alpha_{0}}\right)$.

Now if $\alpha_{0}$ is a long root, the assumption that $\mu=\lambda^{\alpha_{0}}$ lies in $m(\Phi)^{i+1} P_{\alpha_{0}}^{*}$ implies that $\lambda$ belongs to $m(\Phi)^{i+1} P^{*}$, a contradiction since we have assumed that $\lambda$ is not an element of $m(\Phi)^{i+1} P^{*}$.

Hence we can assume that both $\alpha_{0}$ and $\alpha$ are short roots. If $m(\Phi)^{i+1}$ divides $m_{\alpha_{0}}(\lambda)$, then by our assumption on $\mu$, we get that $\lambda \in m(\Phi)^{i+1} P^{*}$, contradicting our initial choice of $\lambda$. Hence, we can write

$$
m_{\alpha_{0}}(\lambda)=m(\Phi)^{i} m^{\prime}
$$

with $m^{\prime}$ coprime to $m(\Phi)$.

Assuming $\mu$ is not of Weyl denominator type, we have a factorization

$$
C(\mu, d(\lambda))=C(\mu, \tilde{d}(\mu) \tilde{\rho}) C(\tilde{d}(\mu) \tilde{\rho}, d(\lambda)),
$$

where $\tilde{d}(\mu)$ is the largest integer $d$ such that $d \tilde{\rho} \mid \mu$. The factor $C(\tilde{d}(\mu) \tilde{\rho}, d(\lambda))$ divides $C(m(\Phi) \tilde{d}(\mu) \rho, d(\lambda))$. In this case, we take

$$
e=m(\Phi) \tilde{d}(\mu), \quad d=d(\lambda) .
$$

We assume that $V$ is not coprime to $C(e \rho, d)$.

If $\mu$ is of Weyl denominator type, then both the factors divide $C(e \rho, d)$, where $e$ is defined as above.

By Proposition 5.1,

$$
\left(m(\Phi) \tilde{d}(\mu), m_{\alpha_{0}}(\lambda)\right)>d .
$$

Since $\mu \in m(\Phi)^{i+1} P_{\alpha_{0}}^{*}$, it follows that $\tilde{d}(\mu)$ is divisible by $m(\Phi)^{i}$. We argue as above and write

$$
\tilde{d}(\mu)=d^{\prime}(\mu) m(\Phi)^{i}, \quad d=d(\lambda)=m(\Phi)^{i} d^{\prime},
$$

where $d^{\prime}$ is coprime to $m(\Phi)$. The inequality given by (5.16) can be written as

$$
\left(m(\Phi) d^{\prime}(\mu), m^{\prime}\right)>d^{\prime} .
$$

On the other hand, Lemma 5.2 yields

$$
d^{\prime}=\left(d(\mu) / m(\Phi)^{i}, m^{\prime}\right) .
$$

Since $\tilde{d}(\mu)$ divides $d(\mu)$, it follows that the only way this is possible is if $m(\Phi)$ divides $m^{\prime}$. This contradicts our choice of $\lambda$.

Hence Theorem 2.3 is proved modulo the proofs of Propositions 5.1 and 5.2 


\section{An ARIthmetic Lemma}

In this section we give an arithmetical argument to establish Proposition 5.1 when the root system is isomorphic to $s l_{2}$. This turns out to be an arithmetic statement in the context of a polynomial ring in one variable over suitable rings. It is this arithmetic statement that is at the heart of the proof of the main theorem in the general case. The proof of the main theorem is to reduce by induction on the rank to the arithmetic statement (and not to the irreducibility of characters, say of $G L(3))$. In retrospect, as irreducibility is connected with divisibility, it is to be expected that the proof of an irreducibility result depends on some arithmetic.

We first recall the following elementary lemma from cyclotomic theory.

Lemma 6.1. Let e be a natural number and let $\zeta_{e}$ denote a primitive e-th root of unity.

i) If e is composite, then $\left(1-\zeta_{e}\right)$ is a unit in the ring $\mathbb{Z}\left[\zeta_{e}\right]$.

ii) If $e=p^{k}$ for some prime number $p$, then $\left(1-\zeta_{e}\right)$ divides $p$ in the ring $\mathbb{Z}\left[\zeta_{e}\right]$. In particular, if $p \mid N$, then $\left(1-\zeta_{e}\right)$ is not a unit in the ring $\mathbb{Z}\left[\zeta_{e}\right]$, and thus in any ring $\mathcal{O}$ containing $\mathbb{Z}\left[\zeta_{e}\right]$ in which $p$ is not invertible.

Proof. Let

$$
\Phi_{e}(x)=\frac{x^{e}-1}{x-1}=1+x+\cdots+x^{e-1} .
$$

We have $\Phi_{e}(1)=e$. Further, if $e$ and $f$ are coprime natural numbers, then $\Phi_{e}$ and $\Phi_{f}$ are coprime polynomials.

For mutually coprime natural numbers $f_{1}, \cdots, f_{k}$ dividing $e$, define

$$
\Phi_{e: f_{1}, \cdots, f_{k}}(x)=\Phi_{e}(x) / \prod_{i=1}^{k} \Phi_{f_{i}}(x)=\prod_{\zeta^{e}=1, \zeta^{f_{i}} \neq 1}(x-\zeta),
$$

where $i$ goes from 1 to $k$ in the above product. The gcd of the coeffecients of $\Phi_{e}(x)$ is 1 , and it is clear that $\prod_{i=1}^{k} \Phi_{f_{i}}(x)$ divides $\Phi_{e}(x)$ in the ring $\mathbb{Q}[x]$. Hence by Gauss's lemma, $\Phi_{e: f_{1}, \cdots, f_{k}}(x) \in \mathbb{Z}[x]$.

Let $e=p_{1}^{r_{1}} \cdots p_{k}^{r_{k}}$ be the factorization of $e$ into primes, where $p_{i} \neq p_{j}$ are mutually distinct rational primes. Since $\Phi_{e: p_{1}^{r_{1}}, \ldots, p_{k}^{r_{k}}}(1)=1$, i) follows from the

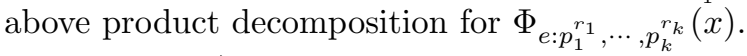

Similarly, ii) follows from the fact that

$$
\Phi_{p^{k}: p^{k-1}}(1)=p
$$

Remark 6.1. The proof uses the fact that we are working over a characteristic zero ring.

The basic arithmetic proposition is the following:

Proposition 6.1. Let e, $f$ be natural numbers not bigger than $N$ and divisible by a natural number $d$. Let $\mathcal{O}$ be a ring satisfying property $\mathbf{A}_{N}$. Suppose there are non-unit elements $U, V \in \mathcal{O}[x]$ and elements $X, Y$ in $\mathcal{O}[x]$ satisfying the following:

$$
\begin{aligned}
U V & =\Phi_{e: d}, \\
U X+V Y & =\Phi_{f: d} .
\end{aligned}
$$

Then the greatest common divisor $(e, f)$ of $e$ and $f$ is strictly greater than $d$. 
Proof. For a natural number $e$ let $\mu_{e}$ denote the group of $e$-th roots of unity; for a rational prime $p$ let $\mu_{p^{\infty}}$ denote the group of roots of unity of order a power of $p$. By enlarging $\mathcal{O}$ by adjoining roots of unity, we can assume that $U$ and $V$ factorizes into linear factors in $\mathcal{O}[x]$. Given a polynomial $W \in \mathcal{O}[x]$, let $Z_{W}$ denote the zeros of $W$ in $\mathcal{O}$. Up to units in $\mathcal{O}[x]$, there is a factorization

$$
U(x)=\prod_{\zeta \in Z_{U}}\left(1-\zeta^{-1} x\right), \quad V(x)=\prod_{\zeta \in Z_{V}}\left(1-\zeta^{-1} x\right),
$$

so that

$$
Z_{U} \cup Z_{V}=\mu(e, d):=\left\{\zeta \mid \zeta^{e}=1, \zeta^{d} \neq 1\right\} .
$$

We first make the following claim:

Claim. There exists $\gamma \in Z_{U}$ (or $Z_{V}$ ) and $\delta \in Z_{V}$ (resp. $\delta \in Z_{U}$ ) such that $\gamma^{-1} \delta \neq 1$ is an element of prime power order for some rational prime $p$.

Proof of Claim. Choose an element $\gamma \in Z_{U}$, such that $\gamma$ can be expressed as a product $\gamma=\gamma_{1} \cdots \gamma_{k}$ having the following properties:

- $\gamma_{i} \in \mu_{p_{i}^{\infty}}$ for some rational primes $p_{i}$.

- $p_{i} \neq p_{j}$ for $1 \leq i, j \leq k$.

- $k$ is minimal amongst all $\gamma \in Z_{U}$.

Since $Z_{U}$ is non-empty, such a choice is possible by the Chinese remainder theorem.

Suppose for some $i, \delta:=\gamma \gamma_{i}^{-1} \in Z_{V}$. Since $\gamma_{i}$ is $p_{i}$-primary,

$$
\gamma^{-1} \delta=\gamma_{i}^{-1}
$$

is $p_{i}$-primary, and this proves the Claim in this case.

Hence we can assume that for any $i=1, \cdots, k, \gamma \gamma_{i}^{-1} \in Z_{U} \cup \mu_{d}$. But since $k$ is minimal, this implies that

$$
\gamma \gamma_{i}^{-1} \in \mu_{d}
$$

for every $i=1, \cdots, k$. If $k>1$, then this implies that $\gamma \in \mu_{d}$, contradicting the fact that $Z_{U}$ is coprime to $\Phi_{d}$. Hence we see that there exists an element $\gamma_{p} \in \mu_{p^{\infty}} \cap Z_{U}$ for some rational prime $p$.

Arguing similarly with $Z_{V}$, we obtain an element $\delta_{q} \in \mu_{q \infty} \cap Z_{V}$ for some rational prime $q$.

If $q=p$, then take $\gamma=\gamma_{p}$ and $\delta=\delta_{q}$. This establishes the claim since the polynomial $\Phi_{e}$ is separable; hence $\gamma^{-1} \delta \neq 1$.

Assume now that $p \neq q$. It follows that the element $\gamma_{p} \delta_{q}$ belongs to the set $\mu(e, d)$ defined as in equation (6.3). If it belongs to $Z_{U}$ (resp. $Z_{V}$ ), then the pair $\gamma=\gamma_{p} \delta_{q}$ (resp. $\delta=\gamma_{p} \delta_{q}$ ) and $\delta=\delta_{q}$ (resp. $\gamma=\gamma_{p}$ ) produces the elements as required by the Claim. This proves the Claim.

Now we deduce the proposition from the Claim. Upon substituting $x=\delta$, we get

$$
U(\delta) X(\delta)+V(\delta) Y(\delta)=\Phi_{f, d}(\delta) .
$$

We have $V(\delta)=0$ and

$$
U(\delta)=\prod_{\zeta \in Z_{U}}\left(1-\zeta^{-1} \delta\right) .
$$


By the Claim, there exists a factor of the form $\left(1-\gamma^{-1} \delta\right)$ of $U$, such that $\gamma^{-1} \delta \in \mu_{p^{\infty}}$ for some rational prime $p$. By part ii) of Lemma 6.1 this implies that $U(\delta)$ is not a unit in $\mathcal{O}$. So the left hand side of equation (6.4) is not a unit. Now,

$$
\Phi_{f: d}(\delta)=\prod_{\zeta \in \mu(f, d)}\left(1-\zeta^{-1} \delta\right) .
$$

Suppose $(e, f)=d$. Then for any pair of divisors $e^{\prime}\left|e, f^{\prime}\right| f$ with $d$ dividing $e^{\prime}$ and $f^{\prime}$ and not equal to either $e^{\prime}$ or $f^{\prime}$, the least common multiple of $e^{\prime}$ and $f^{\prime}$ has at least two distinct prime factors. Hence for any $\zeta \in \mu(f, d)$ of order $f^{\prime}$ (take $\delta$ to be of order $\left.e^{\prime}\right)$, the factor $\left(1-\zeta^{-1} \delta\right)$ is a unit. Hence $\Phi_{f, d}(\delta)$ is a unit, contradicting equation (6.4), and this proves the proposition.

Remark 6.2. The proof of Proposition 6.1 given out here uses characteristic zero methods. Consequently, the proof of the absolute irreducibility of characters (Theorem 2.3) given in this paper does not carry over to positive characteristics, even for those admissible weights for which the Weyl character formula is known to be valid.

For the root system given by $s l_{2}$, the fundamental weight is given by $\rho$. The ring $\mathbb{C}[P]$ can be identified with the ring of Laurent polynomials $\mathbb{C}\left[x, x^{-1}\right]$ by substituting $x=e^{\rho}$. For any pair of natural numbers $e, d$ with $d \mid e$, the element $C(e \rho, d)$ can be written as

$$
C(e \rho, d)=\frac{x^{e}-x^{-e}}{x^{d}-x^{-d}}=x^{e-d} \frac{x^{2 e}-1}{x^{2 d}-1}=x^{e-d} \Phi_{2 e, 2 d} .
$$

Corollary 6.1. Proposition 5.1 is valid for the root system given by the Lie algebra $s l_{2}$, i.e., let $e, c, f=e+c$ be natural numbers and let $\mu=e \rho, \eta=f \rho=(e+c) \rho$ be dominant weights for the root system $A_{1}$. Let $\mathcal{O}$ be a ring satisfying property $\mathbf{A}_{2 N}$, for some natural number $N$ larger than $e$ and $f$. Let $d$ be a natural number dividing e, c. Suppose there are non-unit elements $U, V \in \mathcal{O}\left[x, x^{-1}\right]$ and elements $X, Y$ in $\mathcal{O}\left[x, x^{-1}\right]$ such that the following pair of equations is satisfied:

$$
\begin{aligned}
U X+V Y & =C(f \rho, d), \\
C(e \rho, d) & =U V .
\end{aligned}
$$

Then $(e, c)=(e, f)>d$.

Proof. From Proposition 6.1 and by equation (6.5), we get $(2 e, 2 f)=2(e, f)>2 d$. Hence it follows that $(e, f)>d$.

Remark 6.3. We do not actually require that $U$ and $V$ are $W$-invariant in the above corollary. The invariant hypothesis is required in the induction step of the proof of Proposition 5.1 at the stage when we use Proposition 5.2.

\section{Proof of Proposition 5.1}

In this section we give a proof of Proposition 5.1 for a simple based root system $\mathcal{R}=(E, \Phi, \Delta)$ of rank $l<r$, needed for the proof of the main theorem for a simple root system of rank $r$. In the rank one case, Proposition 5.1 is proved as Corollary 6.1 in the last section using arithmetic methods. In contrast, the proof in the higher rank case proceeds by induction on the rank; we reduce to a lower rank situation using Proposition 5.2 In this section, we will be implicitly working over a ring $\mathcal{O}$ satisfying property $\mathbf{A}_{N}$, since it is required for the initial induction step given by 
Corollary 6.1, but we don't need the arithmetical properties of the ring $\mathcal{O}$ in this section.

A fundamental ingredient in the proof of Proposition 5.1 is the "universal divisibility' of the Weyl denominator as in Proposition 2.1. This is applied in the following manner:

Lemma 7.1. Let $d$ be a natural number and let $U$ be a factor of either $S(d \rho)$ or $S(d \tilde{\rho})$. Suppose $\alpha$ is a corner root. Consider the cofactor expansion of $U$ along $\alpha$,

$$
U=\sum_{i \geq 0} e^{(u-i) l_{\alpha}} U_{\alpha, u-i}
$$

Then the leading coefficient $U_{\alpha, u}$ divides $U_{\alpha, u-i}$ for all $i \geq 0$.

Proof. It is enough, by duality, to prove the lemma when $U$ is a factor of $S(d \rho)$. The leading coefficient of $S(\rho)$ along $\alpha$ is given by $S\left(\rho^{\alpha}\right)$, which is the Weyl denominator for the root system $\mathcal{R}_{\alpha}$. By universal divisibility of the Weyl denominator, $S\left(\rho^{\alpha}\right)$ divides all the coefficients occurring in the cofactor expansion along $\alpha$ of $S(\rho)$. Scaling by $d$ implies that the leading coefficient $S\left(d \rho^{\alpha}\right)$ of $S(d \rho)$ along $\alpha$ divides all the coefficients in the cofactor expansion of $S(d \rho)$ along $\alpha$. From the separability of $S\left(d \rho^{\alpha}\right)$ and applying the proof of the Eisenstein criterion, the lemma follows for any factor $U$ of $S(d \rho)$.

We now begin the proof of Proposition 5.1. The proposition is true for the rank one root system by Corollary 6.1. By induction, assume that the proposition holds for all simple root systems of rank less than $l$. By Proposition 5.2 (this is where we use the fact that the factors are $W$-invariant; see also Remark 7.1 at the end of this section) there is a corner root $\beta$ of the Dynkin diagram of $\Phi$ such that the leading coefficient $U_{\beta, u}$ (resp. $V_{\beta, v}$ ) of $U$ (resp. $V$ ) in the cofactor expansion along $\beta$ is not monic.

Suppose $\beta \neq \alpha$. The root $\alpha$ considered as an element in the root system $\mathcal{R}_{\beta}$ continues to have the same property regarding length, either short or long, as $\alpha$ has. We have

$$
\eta^{\beta}=\mu^{\beta}+c \omega_{\alpha}^{\beta}
$$

where $\omega_{\alpha}^{\beta}$ now denotes the fundamental weight corresponding to $\alpha$ for the root system $\mathcal{R}_{\beta}$. In particular, this yields, $N \geq N(\eta) \geq N\left(\eta^{\beta}\right)$. Comparing the leading terms in equations (5.1) and (5.2), we get

$$
\begin{aligned}
C\left(\mu^{\beta}, d\right) & =U_{\beta, u} V_{\beta, v}, \\
U_{\beta, u} X_{\beta, x}+V_{\beta, v} Y_{\beta, y} & =C\left(\eta^{\beta}, d\right),
\end{aligned}
$$

where $X_{\beta, x}$ and $Y_{\beta, y}$ are the leading coefficients of $X$ and $Y$ respectively. Since the leading coefficient of $C(e \rho, d)$ along $\beta$ is given by $C\left(e \rho^{\beta}, d\right)$ and $V_{\beta, v}$ divides it, we obtain by the induction hypothesis that $(e, c)>d$ if either $\mathcal{R}_{\beta}$ is simply laced or $\alpha$ is a short root, and $\left(e, m\left(\Phi_{\beta}\right) c\right)>d$ if $\mathcal{R}_{\beta}$ is not simply laced and $\alpha$ is a long root. This establishes Proposition 5.1, since $m\left(\Phi_{\beta}\right)=m(\Phi)$ if $m\left(\Phi_{\beta}\right)$ is non-trivial.

Now suppose $\beta=\alpha$. In this case, we get

$$
\eta^{\alpha}=\mu^{\alpha} \quad \text { and } \quad C\left(\mu^{\alpha}, d\right)=C\left(\eta^{\alpha}, d\right)=U_{\alpha, u} V_{\alpha, v},
$$

and it looks as if it is impossible to set up the inductive process. The trick out here is to observe that the universal divisibility of the Weyl denominator, together with 
the fact that $V$ is of Weyl denominator type, allow us to perform the inductive step by considering the expansion up to the second leading non-zero term of $S(\eta)$.

Considering the leading terms in equations (5.1) and (5.2), we get the following equations:

$$
\begin{aligned}
& C\left(\eta^{\alpha}, d\right)=U_{\alpha, u} V_{\alpha, v}, \\
& C\left(\eta^{\alpha}, d\right)=U_{\alpha, u} X_{\alpha, x}+V_{\alpha, v} Y_{\alpha, y} .
\end{aligned}
$$

The separability of $C\left(\eta^{\alpha}, d\right)$ implies that $U_{\alpha, u}$ and $V_{\alpha, v}$ are coprime. Hence the above equations imply that $V_{\alpha, v}$ divides $X_{\alpha, x}$.

We continue comparing the coefficients in the cofactor expansion along $\alpha$ of the equation

$$
S(\eta)=(U X+V Y) S(d \rho) .
$$

Let $T=S(d \rho)$. Denote by $s$ (resp. $u, v, x, y, t$ ) the degrees of $S(\eta)$ (resp. $U, V, X, Y, T)$ with respect to the cofactor expansion along $\alpha$. Here $s=\omega_{\alpha}^{*}(\eta)$, and we have

$$
\omega_{\alpha}^{*}(\eta)-\omega_{\alpha}^{*}\left(s_{\alpha}(\eta)\right)=m_{\alpha}(\eta) .
$$

Upon equating the $l_{\alpha}^{s-a}$-degree term in the cofactor expansion of $S(\eta)$ along $\alpha$, we get

$$
\sum_{i+j+k=a} U_{u-i} X_{x-j} T_{t-k}+\sum_{l+m+n=a} V_{v-l} Y_{y-m} T_{t-n}=S(\eta)_{s-a}
$$

where we have suppressed the use of the subscript $\alpha$. The coefficients $S(\eta)_{s-a}$ of the $l_{\alpha}^{s-a}$-degree term in the cofactor expansion of $S(\eta)$ along $\alpha$ are given by

$$
S(\eta)_{s-a}= \begin{cases}S\left(\eta^{\alpha}\right) & \text { if } a=0, \\ 0 & \text { if } 0<a<m_{\alpha}(\eta), \\ S\left(\left(s_{\alpha} \eta\right)^{\alpha}\right) & \text { if } a=m_{\alpha}(\eta) .\end{cases}
$$

To start the induction, we have that $V_{v}$ divides $X_{x}$. By induction assume that $V_{v}$ divides $X_{x-j}$ for $j<a$. Now suppose $0<a<m_{\alpha}(\eta)$ The right hand side in equation (7.3) is zero, and equation (7.3) gives

$$
0=X_{x-a} U_{u} S\left(d \rho^{\alpha}\right)+\sum_{i+j+k=a, i>0} U_{u-i} X_{x-j} T_{t-k}+\sum_{l+m+n=a} V_{v-l} Y_{y-m} T_{t-n} .
$$

Since $V$ is of Weyl denominator type, by Lemma 7.1 the leading coefficient $V_{v}$ divides all the coefficients $V_{v-j}$ for all $j \geq 0$ in the cofactor expansion of $V$ along $\alpha$. Further, in the second sum on the right, the assumption that $i>0$ implies that $j<a$. Hence by induction and the fact that $V_{v}$ is coprime to $S\left(d \rho^{\alpha}\right)$ and $U_{u}$, we get that $V_{v}$ divides $X_{x-a}$ for $a<m_{\alpha}(\eta)$.

Thus upon comparing the $s-a=m_{\alpha}(\eta)$ term in equation (7.3) we get

$$
\left(U_{u} X_{x-a}+V_{v} \tilde{Y}\right) S\left(d \rho^{\alpha}\right)=S\left(\left(s_{\alpha} \eta\right)^{\alpha}\right),
$$

for some element $\tilde{Y} \in \mathcal{O}\left[P^{\alpha}\right]$. Here we have used the fact that the leading coefficient $S\left(d \rho^{\alpha}\right)$ divides all the other coefficients $T_{t-j}$ in the cofactor expansion of $S(d \rho)$. Now, by equation (4.12),

$$
\left(s_{\alpha} \eta\right)^{\alpha}=\eta^{\alpha}+c_{n} \omega_{\alpha_{n}}^{\alpha},
$$


where $\alpha_{n}$ is the unique element of $\Delta$ that is connected to the corner root $\alpha$ (since $\alpha=\beta$ is a corner root). The value of $c_{n}$ is given by

$$
\begin{aligned}
c_{n} & =\left|\left\langle\alpha_{n}^{*}, \alpha\right\rangle\right| m_{\alpha}(\eta) \\
& = \begin{cases}m(\Phi)\left(m_{\alpha}(\mu)+c\right) & \text { if } \alpha \text { is long and } \alpha_{n} \text { is short, } \\
m_{\alpha}(\mu)+c & \text { otherwise. }\end{cases}
\end{aligned}
$$

Summing up, we have the following equations given by equation (7.1) and equation (7.5):

$$
\begin{aligned}
U_{\alpha, u} V_{\alpha, v} & =C\left(\eta^{\alpha}, d\right), \\
U_{\alpha, u} X_{x-a}+V_{\alpha, v} \tilde{Y} & =C\left(\eta^{\alpha}+c_{n} \omega_{\alpha_{n}}^{\alpha}, d\right) .
\end{aligned}
$$

The projection $\omega_{\alpha_{n}}^{\alpha}$ of the fundamental weight corresponding to $\alpha_{n}$ in $\mathcal{R}$ is the fundamental weight corresponding to the corner root $\alpha_{n}^{\alpha}$ in the root system $\mathcal{R}_{\alpha}$.

We apply the induction hypothesis to the root system $\mathcal{R}_{\alpha}$ and the equations given by equation (7.8). Again, it is easy to see that

$$
N \geq N(\eta) \geq N\left(\eta^{\alpha}+c_{n} \omega_{\alpha_{n}}^{\alpha}\right) .
$$

Note that by assumption $e$ divides $d(\mu)$; in particular, $d \mid m_{\alpha}(\mu)$. If $\alpha$ is long and $\alpha_{n}$ is short, then the root system $\mathcal{R}_{\alpha}$ is simply laced. By the inductive hypothesis, we obtain

$$
d<\left(e, c_{n}\right)=\left(e, m(\Phi)\left(m_{\alpha}(\mu)+c\right)\right)=(e, m(\Phi) c),
$$

establishing Proposition 5.1 in this case. Note that equation (7.7) implies that $N\left(\left(s_{\alpha} \eta\right)^{\alpha}\right) \leq N(\eta) \leq N$.

If $\mathcal{R}_{\alpha}$ is not simply laced and $\alpha_{n}$ is a long root, the inductive hypothesis again yields

$$
d<\left(e, m\left(\Phi_{\alpha}\right) c_{n}\right)=\left(e, m\left(\Phi_{\alpha}\right)\left(m_{\alpha}(\mu)+c\right)\right)=(e, m(\Phi) c)
$$

since $m\left(\Phi_{\alpha}\right)=m(\Phi)$.

Finally, if either $\mathcal{R}_{\alpha}$ is simply laced or $\alpha_{n}^{\alpha}$ is a short root in $\mathcal{R}_{\alpha}$, then the inductive hypothesis gives

$$
d<\left(e, c_{n}\right)=\left(e, m_{\alpha}(\mu)+c\right)=(e, c) .
$$

This proves Proposition 5.1

Remark 7.1. One can avoid the use of Proposition 5.2 in this proof by trying to directly prove the required statement. Indeed, in the simply laced case, the leading coefficients of a non-constant invariant $V$ dividing a generalized Weyl denominator function $S(d \rho)$ can easily be seen to be non-trivial along any corner root. It is here that we require the factor $V$ to be invariant. For the other term $U$, if it contains a factor not of Weyl denominator type, it is not too difficult to see that there has to be at least one corner root along which the leading coefficient is a non-unit. It seems possible to extend this argument to cover the non-simply laced cases too, and avoid using the more general Proposition 5.2 .

\section{NON-EXISTENCE OF INVARIANT MONIC FACTORIZATIONS}

Our aim in this section is to prove the non-existence of monic factorizations given by Proposition 5.2 
8.1. $G L(r)$. We first give the proof for $G L(r)$. We restate Proposition 5.2 in the context of $G L(r)$ :

Proposition 8.1. Let $\lambda=\left(a_{1}, \cdots, a_{r-1}, 0\right)$ be a normalized highest weight for $G L(r)$. Suppose there is a factorization

$$
C(\lambda, d(\lambda))=U V, \quad U, V \in \mathbb{C}\left[x_{1}, \cdots, x_{r}\right]^{W} .
$$

Write

$$
\begin{gathered}
U\left(x_{1}, \cdots, x_{r}\right)=x_{1}^{u} U_{u}+x_{1}^{u-1} U_{u-1}+\cdots+\left(x_{2} \cdots x_{r}\right)^{u_{0}} U_{0}, \\
V\left(x_{1}, \cdots, x_{r}\right)=x_{1}^{v} V_{v}+x_{1}^{v-1} V_{v-1}+\cdots+\left(x_{2} \cdots x_{r}\right)^{v_{0}} V_{0},
\end{gathered}
$$

where $U_{u}, \cdots, U_{0}$ and $V_{v}, \cdots, V_{0}$ are polynomials in the variables $x_{2}, \cdots, x_{r}$, and $U_{0}, V_{0}$ are coprime to $x_{2} \cdots x_{r}$. Then either $U_{u}$ and $V_{v}$ or $U_{0}$ and $V_{0}$ are both non-constant polynomials.

Proof. We use the notation as given in Section 4 for the cofactor expansion of $G L(r)$. Let $d=d(\lambda)$. We have

$$
U V S(d \rho)=S(\lambda)
$$

Suppose $C\left(\lambda^{(1)}, d\right)$ and $C\left(\lambda^{(r-1)}, d\right)$ are both constant polynomials. Then $\lambda=d \rho$ and there is nothing to prove. We assume that $C\left(\lambda^{(1)}, d\right)$ is non-constant (and a similar argument can be given if we assume that $C\left(\lambda^{(r-1)}, d\right)$ is non-constant).

Suppose $V_{v}$ is constant. By $W$-invariance,

$$
V_{0}=\left(x_{2}^{v}+\cdots+x_{r}^{v}\right)+\text { lower degree terms. }
$$

Hence $V_{0}$ is non-constant. If $U_{0}$ is also non-constant, then we are done. Hence we can assume that $U_{0}$ is constant. By symmetry, this implies that the leading coefficient $U_{u}$ is not constant. Then,

$$
v \geq \operatorname{deg}_{x_{2}}\left(V_{0}\right)=\operatorname{deg}_{x_{2}}\left(C\left(\lambda_{0}, d\right)\right)=\left(a_{1}-a_{r-1}\right)-d(r-2) .
$$

Since $S_{\lambda^{(1)}}$ is separable by Corollary 9.2 , applying the Eisenstein criterion we get that $U_{u}$ divides $U_{u}, \cdots, U_{u-\left(a_{1}-a_{2}\right)+1}$. Now if $u-\left(a_{1}-a_{2}\right)+1 \leq 0$, this implies $U_{u}$ divides $U$, and hence $U_{u}$ divides $S_{\lambda^{(2)}}$. But by Corollary $9.4, U_{u}=C\left(\lambda^{(1)}, d\right)$ is coprime to $C\left(\lambda^{(2)}, d\right)$, and this leads to a contradiction. Hence,

$$
u \geq a_{1}-a_{2} .
$$

Since $u+v+d(r-1)=a_{1}$, we get

$$
a_{1}-a_{2} \leq u=a_{1}-v-d(r-1)=a_{r-1}-d .
$$

Similarly arguing with the constant term, since $V_{0}$ is non-constant, we get from the Eisenstein criterion that $V_{0}$ divides $V_{0}, \cdots, V_{a_{r-1}-1}$. By the coprimality of $C\left(\lambda^{(r-1)}, d\right)$ and $C\left(\lambda^{(r-2)}, d\right)$, we get

$$
v \geq a_{r-1}
$$

Hence

$$
u \leq\left(a_{1}-d(r-1)\right)-a_{r-1}=a_{1}-a_{r-1}-d(r-1) .
$$

But

$$
\operatorname{deg}_{x_{2}}\left(U_{u}\right)=\operatorname{deg}_{x_{2}}\left(C\left(\lambda^{(1)}, d\right)\right)=a_{2}-d(r-2) .
$$

By $W$-equivariance,

$$
u \geq a_{2}-d(r-2) .
$$


Hence we get

$$
\begin{gathered}
a_{2}-d(r-2) \leq a_{1}-a_{r-1}-d(r-1), \\
a_{r-1} \leq\left(a_{1}-a_{2}\right)-d .
\end{gathered}
$$

Combining the above inequalities, we get

$$
a_{r-1} \leq\left(a_{1}-a_{2}\right)-d \leq a_{r-1}-2 d,
$$

clearly a contradiction. This proves the proposition.

8.2. Proof for $D, E$. The proof of Proposition 5.2 is easiest for the root systems of type $D, E$ since there are more than two corner roots. We first observe a simple fact about polynomial rings $R[x]$ in one variable over an integral domain $R$ : the degree of a polynomial is the sum of the degrees of its factors. Applying this to the ring $\mathbb{C}[P]^{W}$, since $\lambda$ has maximal degree in the cofactor expansion along any root $\alpha \in \Delta$, amongst all the weights occurring in $S(\lambda)$, we have:

Lemma 8.1. Suppose $S(\lambda)=U V S(d(\lambda) \rho)$, where $U$ and $V$ are $W$-invariant. Assume that there are weights $\mu \in P(U), \nu \in P(V)$ such that

$$
\lambda-d(\lambda) \rho=\mu+\nu .
$$

Then $\mu$ (resp. $\nu)$ has maximal degree in $P(U)$ (resp. $P(V)$ ) with respect to any corner root in $\Delta$.

In particular, if $U$ is monic along $\alpha$, then $\mu$ is the unique weight in $P(U)$ having maximal degree along $\alpha$ and $\mu=u \omega_{\alpha}$ for some integer $u$.

The last assertion that $\mu$ is a multiple of $\omega_{\alpha}$ follows from the fact that $\mu$ is fixed by the group $W_{\alpha}$ since $U$ is $W$ (hence $W_{\alpha}$ )-invariant.

Corollary 8.1. Suppose $U$ is an invariant factor of $S(\lambda)$ which is monic along a corner root $\alpha$. Then $U$ cannot be monic along a different corner root $\beta$.

Indeed, the unique 'highest weight' $\mu \in P(U)$ cannot simultaneously be a multiple of $\omega_{\alpha}$ and $\omega_{\beta}$. This corollary expresses the fact used in the proof of Proposition 8.1, that if a symmetric homogeneous polynomial in at least two variables is considered monic as a polynomial in one variable, then its constant term cannot be a monomial.

Corollary 8.2. Proposition 5.2 is true for the simple root systems of type $D$ and E.

Proof. Since there are three corner roots for the root systems of type $D, E$, this implies that there is at least one corner root at which both $U$ and $V$ are not monic.

8.3. Non-simply laced root systems. From now on we consider a non-simply laced based root system $\mathcal{R}=(E, \Phi, \Delta)$. By Corollary 8.1, we have

Corollary 8.3. Suppose $S(\lambda)=U V S(d(\lambda) \rho)$, where $U$ and $V$ are $W$-invariant. Assume further that $U$ is monic along one of the corner roots $\alpha$ and $V$ is monic along the other corner root $\beta$ in the Dynkin diagram associated to $\mathcal{R}$. Then,

$$
\lambda=u \omega_{\alpha}+v \omega_{\beta}+d(\lambda) \rho,
$$

with $d(\lambda)=(u+d(\lambda), v+d(\lambda))$. 
Proof. If $U$ is symmetric and monic along a corner root $\alpha$, by Corollary $8.1 U$ cannot be monic along the other corner root, say $\beta$. If $V$ is also monic along $\alpha$, then Proposition 5.2 is true. Hence we can assume that $V$ is monic along $\beta$. Assume that $U$ (resp. $V$ ) has an unique maximal weight $u \omega_{\alpha}$ (resp. $\left.v \omega_{\beta}\right)$. This implies that the weight $\lambda$ can be written as $\lambda=u \omega_{\alpha}+v \omega_{\beta}+d(\lambda) \rho$.

We recall the following fact $[\mathrm{H}$, Exercise 5, Section 13, page 72] and its consequences of relevance to us:

Lemma 8.2. Let $\mathcal{R}=(E, \Phi, \Delta)$ be a simple based root system.

(1) Suppose $\mathcal{R}$ is of type $A_{1}, B_{r}, C_{r}, F_{4}, G_{2}$. Then -1 is an element of the Weyl group of $\mathcal{R}$.

(2) Suppose $\mathcal{R}$ is of type $B_{2}, C_{2}, F_{4}, G_{2}$ and $\alpha$ be any corner root in the Dynkin diagram associated to $\mathcal{R}$. Then -1 is an element of the Weyl group of $\mathcal{R}_{\alpha}$.

(3) If $r \geq 3$, then there is a corner root $\alpha$ in the Dynkin diagram associated to $\mathcal{R}=B_{r}$ (resp. $C_{r}$ ) such that $\mathcal{R}_{\alpha}$ is again of type $B$ (resp. C). In particular, -1 is an element of the Weyl group of $\mathcal{R}_{\alpha}$.

For $\alpha, \beta \in \Delta$, let

$$
w_{\alpha \beta}=\left\langle\omega_{\alpha}^{*}, \omega_{\beta}\right\rangle .
$$

This quantity is independent of the $W$-invariant inner product on $E$. The consequence of the first part of the foregoing lemma is that the weights occurring in $S(\lambda)$ are invariant with respect to the map $p \mapsto-p, p \in E$. An application of the proof of Eisenstein's criterion yields the trivial estimate:

Lemma 8.3. With notation as in Corollary 8.3, the following holds:

$$
2 u w_{\beta \alpha}+1 \geq\left\langle\beta^{*}, \lambda\right\rangle \text {. }
$$

Proof. We have the cofactor expansion of $U$ along $\beta$ :

$$
U=\sum_{j=0}^{2 u w_{\beta \alpha}} e^{\left(u w_{\beta \alpha}-j\right) l_{\beta}} U_{\beta, u w_{\beta \alpha}-j} .
$$

Here we have used the symmetry of $U$, the fact that -1 belongs to the Weyl group, to obtain that the degree along $\beta$ of the weights in $U$ varies from $u w_{\beta \alpha}$ to $-u w_{\beta \alpha}$, since the weight with maximum degree along $\beta$ is given by $u \omega_{\beta}$. From the proof of the Eisenstein criterion, we get that $U_{\beta, u w_{\beta \alpha}}$ divides the terms

$$
U_{\beta, u w_{\beta \alpha}}, \cdots, U_{\beta, u w_{\beta \alpha}-\left\langle\beta^{*}, \lambda\right\rangle+1} .
$$

Consequently, if $2 u w_{\beta \alpha}+1$ is less than $\left\langle\beta^{*}, \lambda\right\rangle$, then $U_{\beta, u w_{\beta \alpha}}$ divides all the coefficients of $U$ along $\beta$, and hence all the coefficients of $S(\lambda)$ along $\beta$. Since $U_{\beta, u w_{\beta \alpha}}$ is not a unit, this contradicts the coprimality of $S\left(\lambda^{\beta}\right)$ and $S\left(\left(s_{\beta} \lambda\right)^{\beta}\right)$ given by Corollary 9.4. Hence the lemma follows.

We can prove a sharper estimate, assuming that -1 belongs to the Weyl group of $\mathcal{R}_{\alpha}$ :

Lemma 8.4. With notation as above, assume that the corner root $\alpha$ is such that the automorphism $x \mapsto-x$ is an element of the Weyl group of the root system $\mathcal{R}_{\alpha}$. Then

$$
v w_{\alpha \beta} \geq\left\langle\alpha^{*}, \lambda\right\rangle \text {. }
$$


Proof. The hypothesis that -1 belongs to the Weyl group of $\mathcal{R}_{\alpha}$ implies that the leading coefficient $S\left(\lambda^{\alpha}\right)$ of $S(\lambda)$ along $\alpha$ is mapped to itself by the inverse map $p \mapsto-p$ of $\mathcal{R}_{\alpha}$. We have the cofactor expansion of $V$ along $\alpha$ :

$$
V=\sum_{j=0}^{2 v w_{\alpha \beta}} e^{v w_{\alpha \beta}-j l_{\alpha}} V_{\alpha, v w_{\alpha \beta}-j .} .
$$

Since $V$ is invariant by $W(\mathcal{R})$ and $-1 \in W(\mathcal{R})$, the term $e^{j l_{\alpha}} V_{\alpha, j}$ goes to the term $e^{-j l_{\alpha}} V_{\alpha,-j}$ by the map $p \mapsto-p$ on $P$. Since $U$ is monic along $\alpha$, the top degree term $V_{\alpha, v w_{\alpha \beta}}$ is equal to $S\left(\lambda^{\alpha}\right) / S\left(d(\lambda) \rho^{\alpha}\right)$. By our hypothesis that -1 belongs to the Weyl group of $\mathcal{R}_{\alpha}$, we find that $V_{\alpha, v w_{\alpha \beta}}=V_{\alpha,-v w_{\alpha \beta}}$. From the proof of Eisenstein's criterion, we have that $V_{\alpha, v w_{\alpha \beta}}$ divides

$$
V_{\alpha, v w_{\alpha \beta}}, \cdots, V_{\alpha, v w_{\alpha \beta}-\left\langle\alpha^{*}, \lambda\right\rangle+1} .
$$

Similarly, since $S(\lambda)$ is symmetric $(-1 \in W)$, we find that $V_{\alpha, v w_{\alpha \beta}}$ divides the coefficients

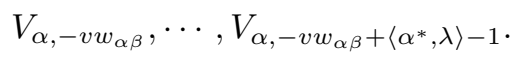

If $v w_{\alpha \beta}$ is less than $\left\langle\alpha^{*}, \lambda\right\rangle$, this implies that $V_{\alpha, v w_{\alpha \beta}}$ divides all the coefficients of $V$ and hence of $S(\lambda)$ in the cofactor expansion of $S(\lambda)$ along $\alpha$. But this contradicts the coprimality of $S\left(\lambda^{\alpha}\right) / S\left(d(\lambda) \rho^{\alpha}\right)$ and $S\left(\left(s_{\alpha} \lambda\right)^{\alpha}\right) / S\left(d(\lambda) \rho^{\alpha}\right)$ given by Corollary 9.4. This establishes the lemma.

We compute the numbers $w_{\alpha \beta}$ explicitly:

Lemma 8.5. Let $\mathcal{R}=(E, \Phi, \Delta)$ be a non-simply laced simple based root system. Let $\alpha, \beta$ be corner roots in the Dynkin diagram associated to $E$. Then the following holds:

(1) $w_{\alpha \beta}>0$.

(2) Let $\mathcal{R}$ be of type $B$ or $C$. Then

$$
w_{\alpha \beta} w_{\beta \alpha}=\frac{1}{2} \text {. }
$$

(3) Let $\mathcal{R}=F_{4}$ or $G_{2}$. Let $\alpha$ (resp. $\beta$ ) be a short (resp. long) corner root of the Dynkin diagram associated to $\mathcal{R}$. Then,

$$
\begin{aligned}
& w_{\alpha \beta}=m(\Phi)= \begin{cases}2 & \text { if } \mathcal{R}=F_{4}, \\
3 & \text { if } \mathcal{R}=G_{2},\end{cases} \\
& w_{\beta \alpha}=1 .
\end{aligned}
$$

Proof. The proof is explicit and case by case. We use the classification of the root systems as given in $\left[\mathrm{H}\right.$, Section 12.1]. For the root systems of type $C_{r}$, take as a base

$$
\alpha=\epsilon_{1}-\epsilon_{2}, \cdots, \epsilon_{r-1}-\epsilon_{r}, 2 \epsilon_{r}=\beta .
$$

The fundamental weights and coweights corresponding to the corner roots are given by

$$
\omega_{\alpha}^{*}=\epsilon_{1} \quad \text { and } \quad \omega_{\beta}^{*}=\left(\sum_{i=1}^{r} \epsilon_{i}\right) / 2 .
$$


Further, $\omega_{\alpha}=\omega_{\alpha}^{*}$ and $\omega_{\beta}=2 \omega_{\beta}^{*}$. Thus,

$$
\begin{aligned}
w_{\alpha \beta} w_{\beta \alpha} & =\left\langle\omega_{\alpha}^{*}, \omega_{\beta}\right\rangle\left\langle\omega_{\beta}^{*}, \omega_{\alpha}\right\rangle \\
& =\frac{1}{2} .
\end{aligned}
$$

For $F_{4}$, we take as a base

$$
\beta=\epsilon_{2}-\epsilon_{3}, \epsilon_{3}-\epsilon_{4}, \epsilon_{4},\left(\epsilon_{1}-\epsilon_{2}-\epsilon_{3}-\epsilon_{4}\right) / 2=\alpha .
$$

Then the fundamental coweights are given by

$$
\omega_{\alpha}^{*}=2 \epsilon_{1} \quad \text { and } \quad \omega_{\beta}^{*}=\epsilon_{1}+\epsilon_{2} .
$$

The fundamental weights are given by $\omega_{\alpha}=\omega_{\alpha}^{*} / 2$ and $\omega_{\beta}=\omega_{\beta}^{*}$. Hence,

$$
\begin{aligned}
& w_{\alpha \beta}=\left\langle\omega_{\alpha}^{*}, \omega_{\beta}\right\rangle=2, \\
& w_{\beta \alpha}=\left\langle\omega_{\beta}^{*}, \omega_{\alpha}\right\rangle=1 .
\end{aligned}
$$

For $G_{2}$, a base is given by

$$
\alpha=\epsilon_{1}-\epsilon_{2}, \beta=-2 \epsilon_{1}+\epsilon_{2}+\epsilon_{3} .
$$

The fundamental coweights are given by

$$
\omega_{\alpha}^{*}=-\epsilon_{2}+\epsilon_{3} \quad \text { and } \quad \omega_{\beta}^{*}=\frac{1}{3}\left(-\epsilon_{1}-\epsilon_{2}+2 \epsilon_{3}\right) .
$$

The fundamental weights are given by $\omega_{\alpha}=\omega_{\alpha}^{*}$ and $\omega_{\beta}=3 \omega_{\beta}^{*}$. Hence,

$$
\begin{aligned}
& w_{\alpha \beta}=\left\langle\omega_{\alpha}^{*}, \omega_{\beta}\right\rangle=3, \\
& w_{\beta \alpha}=\left\langle\omega_{\beta}^{*}, \omega_{\alpha}\right\rangle=1 .
\end{aligned}
$$

This proves the lemma.

8.3.1. Proof of Proposition 5.2 for $B$ and $C$. We now prove Proposition 5.2 for the simple root systems $\mathcal{R}$ of type $B_{r}$ and $C_{r}$. We stick to the above notation. We choose $\alpha$ as in part (3) of Lemma 8.2 , and let $\beta$ be the other corner root of the Dynkin diagram attached to $\mathcal{R}$. Write $\lambda=u \omega_{\alpha}+v \omega_{\beta}+d(\lambda) \rho$ as given by Corollary 8.3. By Lemmas 8.3 and 8.4 we obtain the inequalities

$$
\begin{aligned}
2 u w_{\beta \alpha}+1 & \geq\left\langle\beta^{*}, \lambda\right\rangle=v+d(\lambda), \\
v w_{\alpha \beta} & \geq\left\langle\alpha^{*}, \lambda\right\rangle=u+d(\lambda) .
\end{aligned}
$$

Consequently,

$$
\begin{aligned}
2 u w_{\beta \alpha} w_{\alpha \beta} & \geq(v+d(\lambda)-1) w_{\alpha \beta} \\
& \geq u+d(\lambda)+w_{\alpha \beta}(d(\lambda)-1) .
\end{aligned}
$$

By part (2) of Lemma 8.5, we have $2 w_{\beta \alpha} w_{\alpha \beta}=1$. Hence,

$$
0 \geq d(\lambda)+w_{\alpha \beta}(d(\lambda)-1) .
$$

But this contradicts the positivity of $d(\lambda)$ and $w_{\alpha \beta}$. Hence this proves Proposition 5.2 for the simple root systems of type $B$ or $C$. 
8.3.2. Proof for $F_{4}$ and $G_{2}$. For these simple root systems $\mathcal{R}$, by Lemma 8.2 , the element -1 belongs to the Weyl group of $\mathcal{R}$ as well as to that of $\mathcal{R}_{\alpha}$ for any corner root $\alpha$. Let $\alpha$ denote the short corner root and $\beta$ the long corner root. Write $\lambda=u \omega_{\alpha}+v \omega_{\beta}+d \rho$, and we assume the factorization $S(\lambda)=U V S(d(\lambda) \rho)$ is such that $U$ is monic along $\alpha$ and $V$ is monic along $\beta$. Applying the sharper estimates given by Lemma 8.4, and from part (3) of Lemma 8.5, we get

$$
\begin{aligned}
m(\Phi) v & =v w_{\alpha \beta} \geq\left\langle\alpha^{*}, \lambda\right\rangle=u+d, \\
u & =u w_{\beta \alpha} \geq\left\langle\beta^{*}, \lambda\right\rangle=v+d .
\end{aligned}
$$

If $\lambda$ as above does not satisfy the above equations, then any invariant factorization is non-monic. This proves Proposition 5.2 provided Assumption NMFG is valid.

Remark 8.1. Unfortunately, this proof develops a gap. If we try to look for cofactor expansions (say for $G_{2}$ ) along any other linear form, we require that the top degree term for $S(\lambda)$ is not monic. This would require that the linear form is invariant under a subgroup of the Weyl group as above, and thus up to a Weyl translate is either $\omega_{\alpha}^{*}$ or $\omega_{\beta}^{*}$. Hence we cannot do better.

\section{UNIQUENESS PROPERTY AND CONSEQUENCES}

In this section, we give a proof of the uniqueness result Theorem 2.4, that if $S\left(\lambda_{1}\right) / S\left(\mu_{1}\right)=S\left(\lambda_{2}\right) / S\left(\mu_{2}\right)$, and these quotients are not equal to 1 , then $\lambda_{1}=\lambda_{2}$ and $\mu_{1}=\mu_{2}$. We also establish the separability and coprimality property for the Schur-Weyl sums required in the proof of Proposition 5.2. It will be clear, although we have not done so, that the corresponding statements for $G L(r)$ go through with the same arguments. We begin with a couple of preliminary lemmas.

Lemma 9.1. Suppose $\lambda, \mu$ are weights associated to a simple based root system $\mathcal{R}=(E, \Phi, \Delta)$, such that for two distinct corner roots $\alpha, \beta$ in the Dynkin diagram of $\mathcal{R}$,

$$
\lambda^{\alpha}=\mu^{\alpha} \quad \text { and } \quad \lambda^{\beta}=\mu^{\beta} .
$$

Then $\lambda=\mu$.

Proof. The hypothesis for a particular corner root $\alpha$ implies an equality of the multiplicities $m_{\gamma}(\lambda)=m_{\gamma}(\mu)$ for all $\gamma \in \Delta$ not equal to $\alpha$. Since this happens at two corner roots, the lemma follows.

We now give the proof of Theorem 2.4. By clearing the denominators, the hypothesis can be reformulated as an equality of products of Schur-Weyl elements,

$$
S\left(\lambda_{1}\right) S\left(\mu_{2}\right)=S\left(\lambda_{2}\right) S\left(\mu_{1}\right) .
$$

We want to show that $\lambda_{1}=\lambda_{2}$ or $\mu_{1}$. Assuming that $\lambda_{1} \neq \mu_{1}$ (or equivalently, $\lambda_{2} \neq \mu_{2}$ ), this will show that $\lambda_{1}=\lambda_{2}$ and $\mu_{1}=\mu_{2}$. The proof is by induction on the rank of the root system. Assume first that $\Phi$ is of rank one isomorphic to the root system associated to $\operatorname{sl}(2)$. The hypothesis indicates that

$$
\left(x^{a}-x^{-a}\right)\left(x^{e}-x^{-e}\right)=\left(x^{b}-x^{-b}\right)\left(x^{f}-x^{-f}\right),
$$

for some positive integers $a, b, e, f$, and $a \neq f$. The proposition follows immediately by comparing the roots on both sides. 
Now assume that $\mathcal{R}$ is of rank $r$, and the theorem has been proved for all simple root systems of rank less than $r$. By Lemma 9.1 and the inductive hypothesis, we can conclude the following:

(i) Since there are three corner roots for the simple root systems of type $D$ and $E$, the theorem follows for them.

(ii) We can assume that there is a corner root, say $\alpha$ at which

$$
\lambda_{1}^{\alpha}=\lambda_{2}^{\alpha} \quad \text { and } \quad \mu_{1}^{\alpha}=\mu_{2}^{\alpha},
$$

and another corner root $\beta$, where

$$
\lambda_{1}^{\beta}=\mu_{1}^{\beta} \quad \text { and } \quad \lambda_{2}^{\beta}=\mu_{2}^{\beta} .
$$

Suppose $m_{\alpha}\left(\lambda_{1}\right)<m_{\alpha}\left(\lambda_{2}\right)$. Since for any weight $\lambda, m_{\alpha}(\lambda)=m_{\alpha}\left(\lambda^{\beta}\right)$, we get

$$
m_{\alpha}\left(\lambda_{1}\right)=m_{\alpha}\left(\mu_{1}\right)<m_{\alpha}\left(\lambda_{2}\right)=m_{\alpha}\left(\mu_{2}\right) .
$$

From Lemma 4.2 and Lemma 4.3, the cofactor expansion for a general weight $\lambda$ is given by

$$
S(\lambda)=e^{a_{\alpha, 1}(\lambda) l_{\alpha}} S\left(\lambda^{\alpha}\right)-e^{\left(a_{\alpha, 1}(\lambda)-m_{\alpha}(\lambda)\right) l_{\alpha}} S\left(\left(s_{\alpha} \lambda\right)^{\alpha}\right)+L(\lambda),
$$

where $L(\lambda)$ denotes the terms of degree along $l_{\alpha}$ less than the second highest degree. Comparing the coefficients of the second leading non-vanishing term on both sides of equation (9.1) in the cofactor expansion along $\alpha$, we get

$$
S\left(\left(s_{\alpha} \lambda_{1}\right)^{\alpha}\right) S\left(\mu_{2}^{\alpha}\right)=S\left(\lambda_{2}^{\alpha}\right) S\left(\left(s_{\alpha} \mu_{1}\right)^{\alpha}\right) .
$$

By induction, if $\lambda_{2}^{\alpha}=\mu_{2}^{\alpha}$, together with equation (9.3), we get $\lambda_{2}=\mu_{2}$, contradicting our hypothesis that they are not equal. The other equality gives

$$
\left(s_{\alpha} \lambda_{1}\right)^{\alpha}=\lambda_{2}^{\alpha}=\lambda_{1}^{\alpha} .
$$

But since $\lambda_{1}$ is regular,

$$
\lambda_{1}^{\alpha}=\left(s_{\alpha} \lambda_{1}\right)^{\alpha}=\lambda_{1}^{\alpha}+c \omega_{\alpha_{n}}^{\alpha},
$$

for some positive integer $c$, where $\alpha_{n}$ is the unique root in the Dynkin diagram connected to $\alpha$. This yields a contradiction and proves Theorem 2.4.

\subsection{A coprimality property.}

Lemma 9.2. Let $X$ be a finitely generated free abelian group and $a_{1}, a_{2} \in X$. Then $e^{a_{1}}-1$ and $e^{a_{2}}-1$ are coprime elements in the group algebra $\mathbb{C}[X]$, unless there exist integers $k, l$ different from zero such that $k a_{1}=l a_{2}$.

Proof. Consider the subspace generated by $a_{1}, a_{2}$ in the rational vector space $X_{\mathbb{Q}}=X \otimes \mathbb{Q}$. Suppose they generate a two dimensional vector subspace. Expand $a_{1}, a_{2}$ to a basis $\left\{a_{1}, a_{2}, \cdots, a_{n}\right\}$ of $X_{\mathbb{Q}}$. Writing $x_{i}=e^{a_{i}}, i=1, \cdots, n$, we can identify $\mathbb{C}\left[X_{\mathbb{Q}}\right]$ with the ring of fractional Laurent series in the variables $x_{1}, \cdots, x_{n}$. We claim that the elements $x_{1}-1$ and $x_{2}-1$ are coprime in $\mathbb{C}\left[X_{\mathbb{Q}}\right]$. This follows from considering the degrees of elements along each variable $x_{i}$, where the degree of an element $U \in \mathbb{C}\left[X_{\mathbb{Q}}\right]$ along $x_{i}$ is defined as the difference between the maximum and minimum degrees in $x_{i}$ of the various monomials occurring in $U$. It is clear that the degree of $U V$ is the sum of the degrees of $U$ and $V$. From this it follows that any element dividing both $x_{1}-1$ and $x_{2}-1$ has to be a monomial. 
Corollary 9.1. Let $\mathcal{R}=(E, \Phi, \Delta)$ be a simple based root system, and $\alpha \neq \pm \beta$ be two roots in $\Phi$. Then for any non-zero integers $k, l$, the elements $e^{k \alpha}-1$ and $e^{l \beta}-1$ are coprime in the group algebra $\mathbb{C}[P]$.

In particular, the element $S(d \rho)$ (and by duality $S(d \tilde{\rho})$ ) is a separable element in the ring $\mathbb{C}[P]$ (see part (c) of Proposition 2.1).

Proof. This follows from the previous lemma and the fact that $\alpha$ and $\beta$ are rational multiples of each other in $P \otimes \mathbb{Q}$ precisely when $\alpha= \pm \beta$.

The following corollary is needed to apply the method of Eisenstein criteria given by Lemma 4.4.

Corollary 9.2. Assume that Theorem 2.3 holds for the based simple root system $\mathcal{R}=(E, \Phi, \Delta)$. Then for any dominant regular weight $\mu$, the Schur-Weyl sum $S(\mu)$ is a separable element in $\mathbb{C}[P]^{W}$.

Proof. Theorem 2.3 implies that there is a factorization of

$$
S(\mu)=P Q
$$

in the ring $\mathbb{C}[P]^{W}$, where $P$ is irreducible and $Q$ is of generalized Weyl denominator form. Hence the corollary follows from the previous corollary.

Corollary 9.3. Suppose $e, f$ are natural numbers with $(e, f)=d$. Then the elements $S(e \rho) / S(d \rho)$ and $S(f \rho) / S(d \rho)$ are coprime in the ring $\mathbb{C}[P]$.

Proof. By Corollary 9.1, we need to bother only with the individual factors associated to a root $\alpha$. Substituting $x=e^{\alpha}$ and comparing roots, it is clear that the elements $\left(e^{2 e \alpha}-1\right) /\left(e^{2 d \alpha}-1\right)$ and $\left(e^{2 f \alpha}-1\right) /\left(e^{2 d \alpha}-1\right)$ are coprime in $\mathbb{C}[P]$.

The above corollary combined with the uniqueness result Theorem 2.4 gives us the following:

Proposition 9.1. Let $\mathcal{R}=(E, \Phi, \Delta)$ be a simple based root system, and assume that Theorem 2.3 holds for $\mathcal{R}$. Suppose $\lambda \neq \mu$ are dominant regular weights in $P$. Let $d$ be the greatest common divisor of $d(\lambda)$ and $d(\mu)$. Then $S(\lambda) / S(d \rho)$ and $S(\mu) / S(d \rho)$ are coprime in the ring $\mathbb{C}[P]^{W}$.

Proof. By Theorem 2.3. the factors $C(\lambda)$ and $C(\mu)$ if non-trivial are irreducible. If they coincide, then by the uniqueness result Theorem 2.4, $\lambda=\mu$. Hence we are left with showing that the Weyl denominator type of $S(d(\lambda) \rho) / S(d \rho)$ and $S(d(\mu) \rho) / S(d \rho)$ are coprime, and this follows from Corollary 9.3 by taking $e=d(\lambda)$ and $f=d(\mu)$.

Finally, we deduce a coprimality result needed in the proof of Proposition 5.2 ,

Corollary 9.4. Let $\mathcal{R}=(E, \Phi, \Delta)$ be a simple based root system of rank $r$ and assume that Theorem 2.3 holds for any simple root system of rank less than $r$. Suppose $\lambda$ is a dominant regular weight in $P$, belonging to $m(\Phi)^{i} P \backslash m(\Phi)^{i+1} P^{*}$ for some $i \geq 0$. Let $\alpha$ be a corner root in the Dynkin diagram associated to $\mathcal{R}$. Then the elements $S\left(\lambda^{\alpha}\right) / S\left(d(\lambda) \rho^{\alpha}\right)$ and $S\left(\left(s_{\alpha} \lambda\right)^{\alpha}\right) / S\left(d(\lambda) \rho^{\alpha}\right)$ are coprime in the ring $\mathbb{C}\left[P_{\alpha}\right]^{W_{\alpha}}$.

Proof. The corollary follows from Proposition 9.1 and Lemma 5.2 
9.2. Unique decomposition of tensor products. We now indicate a proof of Theorem 1.1 stated in the introductory section, and our original motivation for establishing an irreducibility result. Unfortunately, since the proof of Theorem 2.3 that we have presented here has a gap, we need to assume Assumption NMFG.

Suppose we have an isomorphism of tensor products,

$$
V_{1} \otimes \cdots \otimes V_{n} \simeq W_{1} \otimes \cdots \otimes W_{m},
$$

as in the hypothesis of Theorem 1.1. Let $\left(\lambda_{i}\right)$ (resp. $\left.\mu_{j}\right)$ denote the highest weight of the irreducible representation $V_{i}$ (resp. $W_{j}$ ). The above hypothesis translates to an equality of products of characters:

$$
\prod_{i=1}^{n} S\left(\lambda_{i}+\rho\right) / S(\rho)=\prod_{j=1}^{m} S\left(\mu_{j}+\rho\right) / S(\rho) .
$$

By the uniqueness result Theorem 2.4 and the irreducibility result Theorem 2.3 , if one of the factors, say $\lambda_{1}+\rho$, is not a multiple of $\rho$, then there exists a $j$ such that $\lambda_{1}+\rho=\mu_{j}+\rho$. Cancelling these factors, we are left with an equality involving fewer characters and we are done.

Hence, we are reduced to the case that each of these weights $\lambda_{i}+\rho$ and $\mu_{j}+\rho$ $(i=1, \cdots, n, j=1, \cdots, m)$ is a multiple of either $\rho$ or $\tilde{\rho}$. By the coprimality result of Corollary 9.1, we have the following equalities for any root $\alpha$ :

$$
\prod_{i=1}^{n} \frac{e^{d_{i} / 2 \alpha}-e^{-d_{i} / 2 \alpha}}{e^{\alpha / 2}-e^{-\alpha / 2}}=\prod_{j=1}^{m} \frac{e^{e_{j} / 2 \alpha}-e^{-e_{j} / 2 \alpha}}{e^{\alpha / 2}-e^{-\alpha / 2}},
$$

for some natural numbers $d_{i}, e_{j}$ (depending also on the relative length of the root). An easy argument using roots of these expressions establishes Theorem 1.1 .

\section{Reduction to the invariant Case For $G L(r)$}

In this section our aim is to extend the irreducibility result for $C(\lambda)$ in the ring $\mathbb{C}[P]^{W}$ given by Theorem 2.3 to the larger ring $\mathbb{C}[P]$, when we are working with $G L(r)$, i.e., to show the irreducibility of $C(\lambda)$ in the polynomial ring $C\left[x_{1}, \cdots, x_{r}\right]$, as claimed in Theorem 1.2 .

Suppose $\lambda$ is a dominant integral weight for $G L(r)$ which is not a multiple of $\rho$. By Lemma 9.1, there is at least one corner root, say $\alpha$, such that the leading coefficient of $C(\lambda)$ along $\alpha$ is not a unit (for $G L(r)$, this is clear: if $C(\lambda)$ is monic as a polynomial in $x_{1}$, then being symmetric the constant term is of the form $\left.x_{2}^{a_{1}-r d(\lambda)}+\cdots+x_{r}^{a_{1}-r d(\lambda)}\right)$.

Suppose $\lambda^{\alpha}$ is not a multiple of $\rho^{\alpha}$. Then there exists a smallest factor $U$ of $C(\lambda)$ in the ring $C\left[x_{1}, \cdots, x_{r}\right]$, such that its leading coefficient along $\alpha$ is divisible by the non-trivial irreducible component $C(\lambda)^{\alpha}$ of the leading coefficient of $C(\lambda)$ along $\alpha$. By construction, $U$ is irreducible, and since the polynomials $S(\mu)$ are separable for any regular weight $\mu, U$ will also be $W_{\alpha}$-invariant.

If $V=C(\lambda) / U$ is monic, then by Lemma 10.1 proved below, both $U$ and $V$ will be symmetric, and the irreducibility result follows by Theorem 2.3 .

On the other hand, if the leading coefficient of $V$ along $\alpha$ is not a unit, then just as in the reduction part of the proof of Theorem 2.3 to that of Proposition 5.1, we have reduced the irreducibility statement to Proposition 5.1 for lower rank. 
Hence, we have reduced to the case that $\lambda^{\alpha}$ is a multiple of $\rho^{\alpha}$ for any corner root $\alpha$. If $r \geq 4$, the rank is at least 3, and this implies that $\lambda$ is a multiple of $\rho$ contradicting our hypthesis on $\lambda$.

Thus we are in the $G L(3)$ situation: if the factor $U$ is irreducible and the quotient $V$ as above is monic, then we are through by Lemma 10.1 as argued above. Otherwise, the leading coefficients of both $U$ and $V$ along $\alpha$ are not units. But in this case, we observe that Proposition 6.1 or Corollary 6.1 is valid without any assumptions of symmetry, and hence the irreduciblity follows as in the reduction of the proof of the main Theorem 2.3 to Proposition 5.1

This proves Theorem 1.2, modulo the following lemma:

Lemma 10.1. With the above notation, let $C(\lambda)=U V$ be a factorization of $C(\lambda)$ in the ring $C\left[x_{1}, \cdots, x_{r}\right]$. Assume further the following: $U$ is irreducible, and $V$ is either monic or the constant coeffecient $V_{0}$ of $V$ is a monomial (considered as a polynomial in $\left.x_{1}\right)$. Then $U$ and $V$ are symmetric polynomials.

Proof. We first observe that if both the leading and constant coeffecients of $C(\lambda, d(\lambda))$ are trivial. then $\lambda^{(1)}=\lambda^{(r-1)}=d \rho$. This implies $\lambda=d \rho$ and so $C(\lambda, d(\lambda))=1$. Hence assume that the leading coeffecient of $C(\lambda, d(\lambda))$ is not monic, and that $V$ is monic. From the proof of Proposition 8.1, we get

$$
u \geq a_{1}-a_{2} .
$$

Since the leading coeffecients of $U$ and $C(\lambda, d(\lambda))$ match, and $U$ is irreducible, we see that $U$ is fixed by the subgroup $S_{r}(1)$ of the symmetric group $S_{r}$ of permutations of the set $1, \cdots, r$ fixing the element 1 . Let $\sigma$ be the transposition in $S_{r}$ interchaning 1 and 2. If $\sigma$ fixes $U$, then since the group generated by $\sigma$ and $S_{r}(1)$ is $S_{r}$, we conclude that $U$ is symmetric. If $U$ is not symmetric, then since $U$ is irreducible, $U^{\sigma}$ must divide $V$. Now the degree in $x_{2}$ variable of $U$ is at least $a_{2}-d$. Hence,

$$
v \geq \operatorname{deg}_{x_{1}} U^{\sigma} \geq \operatorname{deg}_{x_{2}} U \geq a_{2}-d .
$$

This yields,

$$
\operatorname{deg}_{x_{1}}(S(\lambda))=a_{1}=u+v+2 d \geq a_{1}-a_{2}+a_{2}-d+2 d>a_{1},
$$

a contradiction.

\section{ACKNOWLEDGEMENT}

The author sincerely thanks P. Deligne for indicating the extra reducibility that happens for the non-simply laced simple Lie algebras arising from duality (as soon as the author stated the conjecture for $G L(r)$ ). This nipped in the bud the problem of figuring out this extra reducibility in the general case. The author also thanks Vikram Mehta for pointing out the reference to ([FI], KKLV1, [KKLV2]) and to C. DeConcini for the reference to the work of R. Dvornicich and U. Zannier.

Most of this work was done when the author was visiting MPIM at Bonn during 2005-06. Apart from streamlining the proofs, especially the inductive proof of Proposition 5.1, the main new ingredient is the reduction to the symmetric case given in Section 10, which was obtained when the author was visiting Muenster during the summer of 2008, supported by SFB 478. The author gives his sincere thanks to these institutions, to IAS at Princeton where the author visited during the first half of 2007 and to ICTP, Trieste (October 2011) for their excellent hospitality and working environment while the author worked on this and other questions. 


\section{REFERENCES}

[B] Nicolas Bourbaki, Lie groups and Lie algebras. Chapters 4-6, Elements of Mathematics (Berlin), Springer-Verlag, Berlin, 2002. Translated from the 1968 French original by Andrew Pressley. MR1890629 (2003a:17001)

[DZ] R. Dvornicich and U. Zannier, Newton functions generating symmetric fields and irreducibility of Schur polynomials, Adv. Math. 222 (2009), no. 6, 1982-2003, DOI 10.1016/j.aim.2009.06.021. MR2562771 (2011e:05273)

[FI] R. Fossum and B. Iversen, On Picard groups of algebraic fibre spaces, J. Pure Appl. Algebra 3 (1973), 269-280. MR0357396 (50 \#9864)

[FH] William Fulton and Joe Harris, Representation theory, Graduate Texts in Mathematics, vol. 129, Springer-Verlag, New York, 1991. A first course; Readings in Mathematics. MR.1153249 (93a:20069)

[H] James E. Humphreys, Introduction to Lie algebras and representation theory, SpringerVerlag, New York, 1972. Graduate Texts in Mathematics, Vol. 9. MR0323842 (48 \#2197)

[KKLV1] Friedrich Knop, Hanspeter Kraft, and Thierry Vust, The Picard group of a G-variety, Algebraische Transformationsgruppen und Invariantentheorie, DMV Sem., vol. 13, Birkhäuser, Basel, 1989, pp. 77-87. MR1044586

[KKLV2] Friedrich Knop, Hanspeter Kraft, Domingo Luna, and Thierry Vust, Local properties of algebraic group actions, Algebraische Transformationsgruppen und Invariantentheorie, DMV Sem., vol. 13, Birkhäuser, Basel, 1989, pp. 63-75. MR1044585

[R] C. S. Rajan, Unique decomposition of tensor products of irreducible representations of simple algebraic groups, Ann. of Math. (2) 160 (2004), no. 2, 683-704, DOI 10.4007/annals.2004.160.683. MR2123935 (2005j:17009)

Tata Institute of Fundamental Research, Homi Bhabha Road, Bombay, 400 005, India

E-mail address: rajan@math.tifr.res.in 